\title{
Hitting Times of Points and Intervals for Symmetric Lévy Processes
}

\author{
Tomasz Grzywny ${ }^{1} \cdot$ Michał Ryznar $^{2}$
}

Received: 11 September 2015 / Accepted: 4 October 2016 / Published online: 19 November 2016

(C) The Author(s) 2016. This article is published with open access at Springerlink.com

\begin{abstract}
For one-dimensional symmetric Lévy processes, which hit every point with positive probability, we give sharp bounds for the tail function $\mathrm{P}^{x}\left(T_{B}>t\right)$, where $T_{B}$ is the first hitting time of $B$ which is either a single point or an interval. The estimates are obtained under some weak type scaling assumptions on the characteristic exponent of the process. We apply these results to prove sharp two-sided estimates of the transition density of the process killed after hitting $B$.
\end{abstract}

Keywords Symmetric Lévy process · Lévy-Khintchine exponent · Hitting time · Harnack inequality $\cdot$ Dirichlet heat kernel

Mathematics Subject Classification (2010) Primary 60G51; Secondary 60J50 · 60J75

\section{Introduction}

The purpose of this paper is to investigate the distribution of the first hitting time of a point or an interval by a symmetric Lévy process such that $\{0\}$ is regular for itself. Such processes hit points with positive probability. Our main results, under certain regularity assumptions,

The research was supported in part by the National Science Centre (Poland): grant 2014/14/M/ST1/00600

Michał Ryznar

michal.ryznar@pwr.edu.pl

Tomasz Grzywny

tomasz.grzywny@pwr.edu.pl

1 Department of Pure and Applied Mathematics, Wrocław University of Science and Technology, ul. Wyb. Wyspiańskiego 27, 50-370 Wrocław, Poland

2 Department of Pure and Applied Mathematics, Wrocław University of Science and Technology, Wrocław, Poland 
provide sharp estimates of the tail function $\mathbb{P}^{x}\left(T_{0}>t\right), t>0$, where $T_{0}$ is the first hitting time of the point 0 by the process starting from $x$. We further derive similar estimates for the first hitting time of an interval of a given width, under some weak scaling assumption on the characteristic exponent $\psi$ of the process. We also find the asymptotic behaviour of the tail function either for the first hitting time of a point or a compact set under the assumption that the characteristic exponent is regularly varying at zero with index $\delta \geq 1$. The estimates or asymptotics obtained in the paper are expressed in terms of the generalized inverse $\psi^{-1}$ of the characteristic exponent and the compensated potential kernel

$$
K(x)=\int_{0}^{\infty}\left(p_{s}(0)-p_{s}(x)\right) d s, x \in \mathbb{R} .
$$

Here $p_{s}(x), s>0, x \in \mathbb{R}$, is the transition density of the process, which must exist for processes we study. If $\psi$ is comparable with a non-decreasing function we are able to provide sharp estimates of $K$ in terms of the characteristic exponent, so in these cases the estimates become quite explicit and are given in terms of the characteristic exponent and its generalized inverse. For example we show that if $\psi$ has the weak lower scaling property with index $\alpha>1$ (see Preliminaries for the definition) then

$$
\mathbb{P}^{x}\left(T_{0}>t\right) \approx \frac{1}{t \psi^{-1}(1 / t)|x| \psi(1 / x)} \wedge 1, \quad x \in \mathbb{R}, t>0 .
$$

Moreover, we find a similar estimate in the case when $\mathbb{P}^{x}\left(T_{0}>t\right)$ is replaced by the tail function of the first hitting time of an interval (see Theorem 5.3). While in principle, for starting points $x$ far away from the interval, such estimates should follow from the estimates of $\mathbb{P}^{x}\left(T_{0}>t\right)$, but for points close to the boundary of the interval the behaviour of the tail function is not clear. In order to overcome this difficulty we proved and then applied the global Harnack inequality under the weak scaling assumption for $\psi$ (see Theorem 4.5). The Harnack inequality is one of the central topics in the potential theory and the present paper contributes to these studies. Usually the Harnack inequality for Lévy or generally Markov processes is proved under the assumptions which enforce the transience of the process and absolute continuity of its Lévy measure [1, 9, 12, 21, 38]. In our case the process is not only recurrent but point recurrent.

Finally, under the assumption the process is unimodal and $\psi$ has the lower and upper weak scaling property we apply the estimates of the hitting times and derive sharp estimates of $p^{D}$, the transition density (heat kernel) of the process killed after hitting an interval. We show that for $D=(-\infty,-R) \cup(R, \infty), R>0$ we have

$$
p_{t}^{D}(x, y) \approx \mathbb{P}^{x}\left(\tau_{D}>t\right) \mathbb{P}^{y}\left(\tau_{D}>t\right) p_{t}(x-y), \quad t>0, x, y \in D,
$$

with the comparability constant independent of $R$ and where $\tau_{D}$ denotes the first exit time from $D$. The problem of estimating the heat kernel for symmetric Lévy processes has brought a lot of attention recently; see e.g. [6, 8, 14, 15, 27]. Most of the results for unbounded open sets are derived under the assumptions implying the transience of the process. The recurrent processes, except isotropic stable [6], were not explored with regard to heat kernel estimates for exterior sets, and to the best of our knowledge our result is the first one with such generality.

Our approach follows general framework used in most of the works on this subject and requires some exit time estimates. While in the transient case some needed hitting or exit time estimates are quite obvious, here they present the main difficulty. This is done in Propositions 6.1, 6.2 and 6.4, by applying the results from Sections 4 and 5. To see the main 
difference between the transient and recurrent case note that, for $D=(-\infty,-R) \cup(R, \infty)$ and $|x|,|y|>2 R$, we have

$$
p_{t}^{D}(x, y) \approx p_{t}(x-y), t>0,
$$

in the stable transient case $[6,16]$. See also $[8,26]$ for further generalizations if the weak scaling properties imply transience. In the case studied in this paper it is certainly not true since $\mathbb{P}^{x}\left(\tau_{D}>t\right)$ decays to 0 while $t \rightarrow \infty$.

One of the drawbacks is that we deal with one-dimensional processes which are point recurrent. It would be desirable to provide such optimal estimates for one or twodimensional recurrent symmetric Lévy processes, which do not hit points. Unfortunately our approach, based on the nice behaviour of the compensated kernel $K$, will not work in this case.

The distribution of the hitting time of points or compact sets for one-dimensional $\alpha$-stable processes was the subject of studies in several papers [18, 25, 28, 29, 31, 32, 37, 42]. Let $T_{B}$ be the first hitting time of a set $B$. Port in [32] found the asymptotics of $\mathbb{P}^{x}\left(T_{B}>t\right), t \rightarrow \infty$ for a compact set $B$ if $1<\alpha<2$, and for not necessarily symmetric stable processes. The density $f_{x}(t)$ of $T_{x}$ for the symmetric $\alpha$-stable process, $1<\alpha<2$, was found in [42]. For spectrally positive (no negative jumps) $\alpha$-stable process, $1<\alpha<2$, Peskir [31] and Simon [37] found the density $f_{x}(t), x>0$, in a form of a series from which one can derive the asymptotics of $f_{x}(t)$ as $t \rightarrow 0^{+}$or $t \rightarrow \infty$. In a recent paper [28] this type of result was extended to $\alpha$-stable processes, $1<\alpha<2$, having both negative and positive jumps. In [28] the authors derived the Mellin transform of the distribution of $T_{x}$ and then successfully inverted it to obtain the series representation of the density of $T_{x}$.

Relatively little is known about the distribution of hitting times of single points for general Lévy processes. To our best knowledge such explicit results as mentioned above do not exist. Only recently Kwaśnicki [29] studied the distribution of $T_{x}$ for symmetric Lévy processes under certain regularity assumptions on the characteristic exponent of the process. The main result of [29] provides an integral representation of the distribution function of $T_{x}$ in terms of generalized eigenfunctions for the killed semigroup upon hitting $\{0\}$. This representation was then successfully applied in [25] to obtain various asymptotics and estimates of the tail function of $T_{x}$ and its derivatives under further additional regularity assumptions on characteristic exponent and the Lévy measure. Namely it is assumed that the process has completely monotone Lévy density. Comparing our results with those obtained in [25] we remark that our assumptions are much less restrictive, however our approach does not allow to treat the estimates of the density or the higher derivatives of the distribution functions. In a forthcoming paper we provide sharp estimates of the density under the weak upper and lower scaling property for the characteristic exponent for unimodal Lévy processes. Moreover we also treat the hitting distribution of intervals and provide sharp estimates and asymptotics of the tail function, which was not investigated in [25]. We also mention that our methods are more elementary and are based on the estimates of the Laplace transforms of the hitting distributions and various estimates of exit probabilities.

The paper is composed as follows. In Section 2 we recall some basic material regarding one-dimensional symmetric Lévy processes and present some auxiliary results which we use in the sequel. In Section 3 we obtain estimates and asymptotics of the tail function $\mathbb{P}^{x}\left(T_{0}>t\right)$. Section 4 is devoted to the uniform Harnack inequality and boundary behaviour of harmonic functions. These tools we use in Section 5 to prove estimates of the function $\mathbb{P}^{x}\left(T_{[-r, r]}>t\right)$. Section 6 focuses on symmetric unimodal processes with weak global scaling. We use the methods and results of the previous sections to obtain estimates of the Dirichlet heat kernel of a complement of an interval. 


\section{Preliminaries}

Throughout the paper by $c, c_{1} \ldots$ we denote nonnegative constants which may depend on other constant parameters only. The value of $c$ or $c_{1} \ldots$ may change from line to line in a chain of estimates. If we use enumerated $C_{1}, C_{2} \ldots$ then they are fixed constants and usually used in the sequel parts of the paper. Any subsets and real functions considered in the paper are assumed to be Borel measurable. The notion $p(u) \approx q(u), u \in A$ means that the ratio $p(u) / q(u), u \in A$ is bounded from below and above by positive (comparability) constants which may depend on other constant parameters only but does not depend on the set $A$.

We present in this section some basic material regarding one-dimensional symmetric Lévy processes which hit points with non-zero probability. For more detailed information, see $[2,11]$. For questions regarding the Markov and the strong Markov properties, semigroup properties, Schrödinger operators and basic potential theory, the reader is referred to [17] and [4].

In this paper we assume that a Lévy process $X=\left(X_{t}, t \geq 0\right)$ [34], is symmetric. By $v$ we denote its Lévy measure and by $\psi$ its Lévy-Khintchine exponent (symbol). Notice that $v$ and $\psi$ are symmetric as well. Recall that any Lévy measure is a measure such that

$$
\int_{\mathbb{R}}\left(|x|^{2} \wedge 1\right) v(d x)<\infty
$$

If the Lévy measure $v$ is absolutely continuous with respect to the Lebesgue measure, then with a slight abuse of notation, we denote its density by $v$ as well. Since the process is symmetric there is $\sigma \in \mathbb{R}$ such that

$$
\psi(\xi)=\int_{\mathbb{R}}(1-\cos \xi x) v(d x)+\sigma^{2} \xi^{2}, \quad \xi \in \mathbb{R},
$$

and

$$
\mathbb{E} e^{i \xi X_{t}}=e^{-t \psi(\xi)}, \quad \xi \in \mathbb{R} .
$$

For $x \in \mathbb{R}$, by $\mathbb{P}^{x}$ and $\mathbb{E}^{x}$ we denote the distribution and the resulting expectation of the process $x+X$. Obviously $\mathbb{P}^{0}=\mathbb{P}$ and $\mathbb{E}^{0}=\mathbb{E}$. The process $X$ is called unimodal if for any $t>0$ the distribution $p_{t}(d x)$ of $X_{t}$ is unimodal, that is, it is absolutely continuous on $\mathbb{R} \backslash\{0\}$ and its density $p_{t}(x)$ is symmetric on $\mathbb{R}$ and non-increasing on $(0, \infty)$. Unimodal Lévy processes are characterized in [40] by unimodal Lévy measures $v(d x)=v(x) d x=$ $v(|x|) d x$.

The first exit time of an (open) set $D \subset \mathbb{R}^{d}$ by the process $X_{t}$ is defined by the formula

$$
\tau_{D}=\inf \left\{t>0 ; X_{t} \notin D\right\} .
$$

If $F \subset \mathbb{R}$ is a closed set we define the first hitting time $T_{F}$ of $F$ as the first exit time from $F^{c}$. In the case when $F=\{a\}, a \in \mathbb{R}$, we denote $T_{F}=T_{a}$.

In this paper we consider symmetric Lévy processes which have the property that

$$
\int_{0}^{\infty} \frac{1}{1+\psi(x)} d x<\infty .
$$

Note that the above condition implies that $\psi$ is unbounded, so excludes compound Poisson processes and in consequence $\psi(x)>0$ for $x \neq 0$. From [11, Theoreme 7 and Theoreme 8] we infer that for symmetric Lévy processes, which are not compound Poisson, the condition (1) is equivalent to the fact that 0 is regular for the set $\{0\}$ that is

$$
\mathbb{P}^{0}\left(T_{0}=0\right)=1 \text {. }
$$


Moreover (1) guarantees that the distribution of $X_{t}, t>0$, is absolutely continuous and its density $p_{t}(\cdot) \in C^{\infty}(\mathbb{R})$.

In general potential theory a very important role is played by $\lambda$-potential kernels, $\lambda>0$, which are defined as

$$
u^{\lambda}(x)=\int_{0}^{\infty} e^{-\lambda t} p_{t}(x) d t, x \in \mathbb{R} .
$$

If the defining integral above is finite for $\lambda=0$ we call $u^{0}(x)=u(x)$ the potential kernel and then the underlying process is transient.

Under the above assumptions it follows from [2, Corollary II.18 and Theorem II.19] that $h^{\lambda}(x)=\mathbb{E}^{0} e^{-\lambda T_{x}}$ is continuous and

$$
u^{\lambda}(x)=\int_{0}^{\infty} e^{-\lambda s} p_{s}(x) d s=u^{\lambda}(0) h^{\lambda}(x) .
$$

Denote

$$
K^{\lambda}(x)=u^{\lambda}(0)-u^{\lambda}(x)
$$

and

$$
K(x)=\lim _{\lambda \rightarrow 0^{+}} K^{\lambda}(x)=\int_{0}^{\infty}\left(p_{s}(0)-p_{s}(x)\right) d s .
$$

By symmetry and [2, Theorem II.19],

$$
K^{\lambda}(x)=\frac{1}{\pi} \int_{0}^{\infty}(1-\cos x s) \frac{1}{\lambda+\psi(s)} d s .
$$

The monotone convergence theorem implies

$$
K(x)=\frac{1}{\pi} \int_{0}^{\infty}(1-\cos x s) \frac{1}{\psi(s)} d s=\frac{1}{x \pi} \int_{0}^{\infty}(1-\cos s) \frac{1}{\psi(s / x)} d s .
$$

For a number of results below we make the assumption that $K$ is non-decreasing on $(0, \infty)$. We do not know any general criterion which guarantees monotonicity, but it is clear that sufficient conditions are: $\psi(x) / x$ is non-decreasing on $(0, \infty)$ or the process $X$ is unimodal. Another interesting problem is the question if monotonicity of $K$ implies some monotonicity properties of $\psi$.

Define $\kappa \in[0, \infty)$ by

$$
\kappa=\frac{\pi}{\int_{0}^{\infty} \frac{d s}{\psi(s)}}
$$

Lemma 2.1 ([41], Theorem 3.1) We have $\mathbb{P}^{0}\left(T_{x}=\infty\right)=\kappa K(x)$. If $\int_{0}^{\infty} \frac{1}{\psi(s)} d s=\infty$ then for any $x \in \mathbb{R}, \mathbb{P}^{0}\left(T_{x}<\infty\right)=1$.

If $\int_{0}^{\infty} \frac{1}{\psi(s)} d s<\infty$ the process $X$ is transient and it is clear from Lemma 2.1 that its potential kernel satisfies

$$
u(x)=\int_{0}^{\infty} p_{s}(x) d s=\frac{1}{\kappa} \mathbb{P}^{x}\left(T_{0}<\infty\right) .
$$

Proposition 2.2 $K$ is subadditive on $\mathbb{R}$.

Proof Observe that $T_{x+y} \leq T_{x}+T_{x+y} \circ T_{x}$, where o denotes the usual shift operation. By the strong Markov property, for $\lambda>0$,

$$
h^{\lambda}(x+y) \geq h^{\lambda}(x) h^{\lambda}(y), \quad x, y \in \mathbb{R} .
$$


Hence

$$
\begin{aligned}
K(x)+K(y)-K(x+y) & =\lim _{\lambda \rightarrow 0}\left[\left(u^{\lambda}(0)-u^{\lambda}(x)\right)+\left(u^{\lambda}(0)-u^{\lambda}(y)\right)-\left(u^{\lambda}(0)-u^{\lambda}(x+y)\right)\right] \\
& =\lim _{\lambda \rightarrow 0} u^{\lambda}(0)\left[1-h^{\lambda}(x)-h^{\lambda}(y)+h^{\lambda}(x+y)\right] \\
& \geq \lim _{\lambda \rightarrow 0} u^{\lambda}(0)\left[1-h^{\lambda}(x)-h^{\lambda}(y)+h^{\lambda}(x) h^{\lambda}(y)\right] \\
& =\lim _{\lambda \rightarrow 0} u^{\lambda}(0)\left[1-h^{\lambda}(x)\right]\left[1-h^{\lambda}(y)\right] \geq 0 .
\end{aligned}
$$

The fundamental object of the potential theory is the killed process $X_{t}^{D}$ when exiting the set $D$. It is defined in terms of sample paths up to time $\tau_{D}$. More precisely,

$$
\mathbb{E}^{x} f\left(X_{t}^{D}\right)=\mathbb{E}^{x}\left[t<\tau_{D} ; f\left(X_{t}\right)\right], \quad t>0 .
$$

The density function of the transition probability of the process $X_{t}^{D}$ is denoted by $p_{t}^{D}$. We have

$$
p_{t}^{D}(x, y)=p_{t}(y-x)-\mathbb{E}^{x}\left[t>\tau_{D} ; p_{t-\tau_{D}}\left(y-X_{\tau_{D}}\right)\right], \quad x, y \in \mathbb{R}^{d} .
$$

Obviously, we obtain

$$
p_{t}^{D}(x, y) \leq p_{t}(y-x), \quad x, y \in \mathbb{R}^{d} .
$$

$\left(p_{t}^{D}\right)_{t>0}$ is a strongly contractive semigroup (under composition) and shares most of properties of the semigroup $p_{t}$. In particular, it is strongly Feller and symmetric: $p_{t}^{D}(x, y)=$ $p_{t}^{D}(y, x)$. The $\lambda$-potential measure of the process $X_{t}^{D}$ started from $x$ is a Borel measure defined as

$$
G_{D}^{\lambda}(x, A)=\int_{0}^{\infty} e^{-\lambda t} \mathbb{P}^{x}\left(X_{t}^{D} \in A\right) d t,
$$

for any Borel subset $A$ of $\mathbb{R}$. For the Lévy processes explored in the paper their potential measures are absolutely continuous and the corresponding density is $\lambda$-potential kernel of the process $X_{t}^{D}$ and is called $\lambda$-Green function of the set $D$. It is denoted by $G_{D}^{\lambda}$ and we have

$$
G_{D}^{\lambda}(x, y)=\int_{0}^{\infty} e^{-\lambda t} p_{t}^{D}(x, y) d t .
$$

If $\lambda=0$ the corresponding 0 -Green function we simply call the Green function of $D$ and denote $G_{D}(x, y)$.

Another important object in the potential theory of $X_{t}$ is the harmonic measure of the set $D$. It is defined by the formula:

$$
P_{D}(x, A)=\mathbb{E}^{x}\left[\tau_{D}<\infty ; \mathbf{1}_{A}\left(X_{\tau_{D}}\right)\right], \quad A \subset \mathbb{R} .
$$

The density kernel (with respect to the Lebesgue measure) of the measure $P_{D}(x, A)$ (if it exists) is called the Poisson kernel of the set $D$. The relationship between the Green function of $D$ and the harmonic measure is provided by the Ikeda-Watanabe formula [24],

$$
P_{D}(x, A)=\int_{D} v(A-y) G_{D}(x, d y), \quad A \subset(\bar{D})^{c} .
$$

Now we define harmonic and regular harmonic functions with respect to the process $X$. Let $u$ be a Borel measurable function on $\mathbb{R}$. We say that $u$ is harmonic function in an open set $D \subset \mathbb{R}$ if

$$
u(x)=\mathbb{E}^{x} u\left(X_{\tau_{B}}\right), \quad x \in B,
$$


for every bounded open set $B$ with the closure $\bar{B} \subset D$. We say that $u$ is regular harmonic in $D$ if

$$
u(x)=\mathbb{E}^{x}\left[\tau_{D}<\infty ; u\left(X_{\tau_{D}}\right)\right], \quad x \in D .
$$

We note that for any open $D$ the Green function $G_{D}(x, y)$ (if exists) is harmonic in $D \backslash\{y\}$ as a function of $x$. This follows from the strong Markov property and is frequently used in the paper.

The following formula for the Green function of the complement of a point can be found in [41, Lemma 4.1], [19, Theorem 6.1] for recurrent processes and [10, Lemma 4] for stable processes.

Proposition $2.3 G_{\{0\}^{c}}(\cdot, \cdot)$ is jointly continuous on $\mathbb{R} \times \mathbb{R}$. Moreover

$$
G_{\{0\}^{c}}(x, y)=K(x)+K(y)-K(y-x)-K(x) K(y) \kappa .
$$

Proof Recall that for $\lambda>0$ we define $K^{\lambda}(x)=u^{\lambda}(0)-u^{\lambda}(x)$. We have

$$
\begin{aligned}
G_{\{0\}^{c}}^{\lambda}(x, y) & =u^{\lambda}(y-x)-\mathbb{E}^{x} e^{-\lambda T_{0}} u^{\lambda}\left(y-X_{T_{0}}\right)=u^{\lambda}(y-x)-h^{\lambda}(x) u^{\lambda}(y) \\
& =-K^{\lambda}(y-x)+K^{\lambda}(y)+K^{\lambda}(x)-\frac{K^{\lambda}(x) K^{\lambda}(y)}{u^{\lambda}(0)} .
\end{aligned}
$$

Hence by the monotone convergence theorem

$$
G_{\{0\}^{c}}(x, y)=K(x)+K(y)-K(y-x)-K(x) K(y) \kappa .
$$

By the dominated convergence theorem we get continuity of $K$ and $G_{\{0\}}$ as well.

The following observation plays a crucial role in the sequel.

Proposition 2.4 For any $x, y \in \mathbb{R}$ we have

$$
G_{\{0\}^{c}}(x, y) \leq 2[K(x) \wedge K(y)] .
$$

If additionally $K(\cdot)$ is non-decreasing on $(0, \infty)$ then for $x y \geq 0$ we have

$$
G_{\{0\}^{c}}(x, y) \geq K(|x| \wedge|y|)-K(x) K(y) \kappa=K(|x| \wedge|y|) \mathbb{P}^{|x| \vee|y|}\left(T_{0}<\infty\right) .
$$

Proof By subadditivity of $K$ we have

$$
K(y) \leq K(x)+K(y-x) .
$$

Hence

$$
G_{\{0\}^{c}}(x, y) \leq 2 K(x) .
$$

If $K(\cdot)$ is non-decreasing on $(0, \infty)$ then for $y \geq x>0$ we have $K(y)-K(y-x) \geq 0$. Hence

$$
K(x)-K(x) K(y) \kappa \leq G_{\{0\}^{c}}(x, y) .
$$

Lemma 2.5 For any $0<|x|<R<|y|$,

$$
G_{\{0\}^{c}}(x, y)=\mathbb{E}^{x} G_{\{0\}^{c}}\left(X_{\tau_{(-R, R)} \wedge T_{0}}, y\right) .
$$

Proof Let $0<r<|x|<R<R^{\prime}<|y|$. Then by harmonicity of $G_{\{0\}^{c}}(\cdot, y)$ on $\left(-R^{\prime}, 0\right) \cup$ $\left(0, R^{\prime}\right)$ we have

$$
G_{\{0\}^{c}}(x, y)=\mathbb{E}^{x} G_{\{0\}^{c}}\left(X_{\tau_{D_{r, R}}}, y\right),
$$


where $D_{r, R}=(-R,-r) \cup(r, R)$. Proposition 2.4, the dominated convergence theorem, continuity of $G_{\{0\}^{c}}$ and quasi-left continuity of $X$ yield the conclusion when we pass $r \rightarrow$ 0 .

Proposition 2.6 For $|x| \in(0, R)$ we have

$$
\mathbb{E}^{x}\left[\tau_{(-R, R)} \wedge T_{0}\right] \leq 4 R K(x) .
$$

Proof By Proposition 2.4,

$$
\begin{aligned}
\mathbb{E}^{x}\left[\tau_{(-R, R)} \wedge T_{0}\right] & =\int_{-R}^{R} G_{(-R, 0) \cup(0, R)}(x, y) d y \leq \int_{-R}^{R} G_{\{0\}^{c}}(x, y) d y \\
& \leq 4 R K(x) .
\end{aligned}
$$

Proposition 2.7 Let $K$ be non-decreasing on $[0, \infty)$. For $R>0$ and $0<|x|<R$,

$$
\frac{1}{6} \frac{K(x)}{K(R)} \leq \mathbb{P}^{x}\left(\tau_{(-R, R)}<T_{0}\right)
$$

If $\kappa=0$, then additionally

$$
\mathbb{P}^{x}\left(\tau_{(-R, R)}<T_{0}\right) \leq 4 \frac{K(x)}{K(R)}
$$

Proof Let $0<x<R$. By Proposition 2.4, Lemma 2.5 and subadditivity of $K$,

$$
K(x) \mathbb{P}^{2 R}\left(T_{0}<\infty\right) \leq G_{\{0\}^{c}}(x, 2 R)=\mathbb{E}^{x} G_{\{0\}^{c}}\left(X_{\tau_{(-R, R)} \wedge T_{0}}, 2 R\right) \leq 4 K(R) \mathbb{P}^{x}\left(\left|X_{\tau_{(-R, R)} \wedge T_{0}}\right| \geq R\right) .
$$

On the other hand, by Lemma 2.1 and subadditivity of $K$,

$$
\mathbb{P}^{x}\left(\tau_{(-R, R)}<T_{0}\right) \geq \mathbb{P}^{x}\left(T_{0}=\infty\right)=\kappa K(x)=\frac{K(x)}{K(2 R)} \mathbb{P}^{2 R}\left(T_{0}=\infty\right) \geq \frac{1}{2} \frac{K(x)}{K(R)} \mathbb{P}^{2 R}\left(T_{0}=\infty\right),
$$

which combined with the first bound above provide the first estimate. Moreover, if $\kappa=0$ then

$$
2 K(x) \geq G_{\{0\}^{c}}(x, 2 R)=\mathbb{E}^{x} G_{\{0\}^{c}}\left(X_{\tau_{(-R, R)} \wedge T_{0}}, 2 R\right) \geq K(R) \mathbb{P}^{x}\left(X_{\tau_{(-R, R)} \wedge T_{0}} \geq R\right)
$$

and

$$
2 K(x) \geq G_{\{0\}^{c}}(x,-2 R)=\mathbb{E}^{x} G_{\{0\}^{c}}\left(X_{\tau_{(-R, R)} \wedge T_{0}},-2 R\right) \geq K(R) \mathbb{P}^{x}\left(X_{\tau_{(-R, R)} \wedge T_{0}} \leq-R\right) .
$$

Hence

$$
4 K(x) \geq K(R) \mathbb{P}^{x}\left(\left|X_{\tau_{(-R, R)} \wedge T_{0}}\right| \geq R\right)
$$

We also consider the renewal function $V$ of the (properly normalized) ascending ladderheight process of $X_{t}$. The ladder-height process is a subordinator with the Laplace exponent

$$
\kappa(\xi)=\exp \left\{\frac{1}{\pi} \int_{0}^{\infty} \frac{\log \psi(\xi \zeta)}{1+\zeta^{2}} d \zeta\right\}, \quad \xi \geq 0,
$$


and $V(x), x \geq 0$, is its potential measure of the interval $[0, x]$. For $x<0$ we set $V(x)=0$. Silverstein studied $V$ and its derivative $V^{\prime}$ as $g$ and $\psi$ in [36, (1.8) and Theorem 2]. The Laplace transform of $V$ is

$$
\mathcal{L} V(\xi)=\int_{0}^{\infty} V(x) e^{-\xi x} d x=\frac{1}{\xi \kappa(\xi)}, \quad \xi>0 .
$$

For instance, $V(x)=x^{\alpha / 2}$ for $x \geq 0$, if $\psi(\xi)=|\xi|^{\alpha}$ [39, Example 3.7]. The definition of $V$ is rather implicit and properties of $V$ are delicate. In particular the decay properties of $V^{\prime}$ are not yet fully understood. For a detailed discussion of $V$ we refer the reader to [5] and [36]. We have $V(x)=0$ for $x \leq 0$ and $V(\infty):=\lim _{r \rightarrow \infty} V(r)=\infty$. Also, $V$ is subadditive:

$$
V(x+y) \leq V(x)+V(y), \quad x, y \in \mathbb{R} .
$$

It is known that $V$ is absolutely continuous and harmonic on $(0, \infty)$ for $X_{t}$. Also $V^{\prime}$ is a positive harmonic function for $X_{t}$ on $(0, \infty)$, hence $V$ is actually (strictly) increasing. For the so-called complete subordinate Brownian motions [35] $V^{\prime}$ is monotone, in fact completely monotone, cf. [30, Proposition 4.5]. This property was crucial for the development in [15, 27], but in general it fails in the present setting cf. [5, Remark 9]. One of the important features of the function $V^{\prime}$ is the fact that the Green function of $(0, \infty)$ can be written as

$$
G_{(0, \infty)}(x, y)=\int_{0}^{x} V^{\prime}(u) V^{\prime}(y-x+u) d u, \quad 0<x<y .
$$

This follows from [2, Theorem VI.20].

Let $\psi^{*}(x)=\sup _{|u| \leq x} \psi(u), x \geq 0$ be the maximal function of $\psi$. By [23, Theorem 2.7],

$$
\psi(s u) \leq \psi^{*}(s u) \leq 2\left(s^{2}+1\right) \psi^{*}(u), \quad s, u \geq 0 .
$$

Below, in Lemmas 2.8-2.11 we collect useful facts which are true for general symmetric Lévy processes, which are not compound Poisson.

Lemma 2.8 ([5], Proposition 2.4) There is an absolute constant $C_{1} \geq 1$ such that

$$
C_{1}^{-1} \frac{1}{\sqrt{\psi^{*}(1 / r)}} \leq V(r) \leq C_{1} \frac{1}{\sqrt{\psi^{*}(1 / r)}}, \quad r>0 .
$$

Lemma 2.9 ([5], (2.23) and (2.24)) There is an absolute constant $C_{2}$ such that

$$
\mathbb{P}^{0}\left(\left|X_{t}\right| \geq r\right) \leq C_{2} \frac{t}{V^{2}(r)}, \quad r>0
$$

and

$$
v[r, \infty) \leq C_{2} \frac{1}{V^{2}(r)}, \quad r>0 .
$$

Moreover for any $D \subset B_{r}$ and $|x|<r / 2$,

$$
\mathbb{P}^{x}\left(\left|X_{\tau_{D}}\right| \geq r\right) \leq C_{2} \frac{\mathbb{E}^{x} \tau_{D}}{V^{2}(r)}, \quad r>0 .
$$

Lemma 2.10 ([30], Theorem 3.1) There is an absolute constant $C_{3}$ such that for $x>0, t>$ 0 ,

$$
C_{3}\left(\frac{V(x)}{\sqrt{t}} \wedge 1\right) \leq \mathbb{P}^{x}\left(\tau_{(0, \infty)}>t\right) \leq 2\left(\frac{V(x)}{\sqrt{t}} \wedge 1\right)
$$


Lemma 2.11 ([22], Proposition 3.7) There is an absolute constant $C_{4}$ such that for any $x \in(0, R)$,

$$
C_{4} \frac{V(x)}{V(R)} \leq \mathbb{P}^{x}\left(X_{\tau_{(0, R)}} \geq R\right) \leq \frac{V(x)}{V(R)} .
$$

In fact we may take $C_{4}=\frac{C_{3}^{2}}{4}$.

Lemma 2.12 ([7], Lemma 1) Let $f:(0, \infty) \mapsto[0, \infty)$ be non-increasing. Then for $x>0$,

$$
\frac{2}{\pi^{2}} \int_{0}^{\infty}\left[1 \wedge(x r)^{2}\right] f(r) d r \leq \int_{0}^{\infty}(1-\cos (x r)) f(r) d r .
$$

For a continuous non-decreasing function $\phi:[0, \infty) \rightarrow[0, \infty)$, such that $\phi(0)=0$ and $\lim _{s \rightarrow \infty} \phi(s)=\infty$ and define the generalized inverse $\phi^{-1}:[0, \infty) \rightarrow[0, \infty)$,

$$
\phi^{-1}(u)=\inf \{s \geq 0: \phi(s) \geq u\}, \quad 0 \leq u<\infty .
$$

The function $\phi^{-1}$ is non-decreasing and càglàd (left continuous with right-hand side limits). Notice that $\phi\left(\phi^{-1}(u)\right)=u$ for $u \in[0, \infty)$ and $\phi^{-1}(\phi(s)) \leq s$ for $s \in[0, \infty)$. Also, if $\varphi:[0, \infty) \rightarrow[0, \infty), \varphi(0)=0, c>0$ and $c \phi \leq \varphi$, then $\phi^{-1}(u) \geq \varphi^{-1}(c u), u \geq 0$. Below we often consider the (unbounded) characteristic exponent $\psi$ of a symmetric Lévy process with infinite Lévy measure and its maximal function $\psi^{*}$, and denote

$$
\psi^{-1}=\left(\psi^{*}\right)^{-1} \text {. }
$$

This short notation is motivated by the following equality:

$$
\inf \{s \geq 0: \psi(s) \geq u\}=\inf \left\{s \geq 0: \psi^{*}(s) \geq u\right\}, \quad 0 \leq u<\infty .
$$

It is rather natural to assume (relative) power-type behaviour for the characteristic exponent $\psi$ of $X$. To this end we consider $\psi$ as a function on $(0, \infty)$. We say that $\psi$ satisfies the global weak lower scaling condition (WLSC) if there are numbers $\alpha>0$ (called the index of the lower scaling) and $\gamma \in(0,1]$, such that

$$
\psi(\lambda \theta) \geq \gamma \lambda^{\alpha} \psi(\theta) \text { for } \lambda \geq 1, \quad \theta>0 .
$$

In short we write $\psi \in \operatorname{WLSC}(\alpha, \gamma)$ or $\psi \in \mathrm{WLSC}$. The global weak upper scaling condition (WUSC) means that there are numbers $\beta<2$ (called the index of the upper scaling) and $\rho \in[1, \infty)$ such that

$$
\psi(\lambda \theta) \leq \rho \lambda^{\beta} \psi(\theta) \text { for } \lambda \geq 1, \quad \theta>0 .
$$

In short, $\psi \in \operatorname{WUSC}(\beta, \rho)$ or $\psi \in \mathrm{WUSC}$.

We call $\alpha, \gamma, \beta, \rho$ the scaling characteristics of $\psi$ or simply the scalings. In most of our results we assume only the lower scaling condition.

Here are further remarks from [7]: We have $\psi \in \operatorname{WLSC}(\alpha, \gamma)$ if and only if $\psi(\theta) / \theta^{\alpha}$ is comparable to a non-decreasing function on $(0, \infty)$, and $\psi \in \operatorname{WUSC}(\gamma, \rho)$ if and only if $\psi(\theta) / \theta^{\beta}$ is comparable to a non-increasing function on $(0, \infty)$, see [7, Lemmas 8,9 and 11$]$.

We are thus led to the behavior of $\psi^{-1}$.

Lemma 2.13 If $\psi \in W L S C(\alpha, \gamma)$, then

$$
\psi^{-1} \in \operatorname{WLSC}\left(1 / 2,\left(\gamma / 48^{2}\right)^{1 / \alpha}\right) \cap \operatorname{WUSC}\left(1 / \alpha,\left(48^{3} / \gamma^{2}\right)^{1 / \alpha}\right) .
$$


Proof The proof is similar to the proof of [7, Lemma 18], where unimodal Lévy processes were considered. Let $W(x)=\int_{\mathbb{R}}\left(1 \wedge(x s)^{2}\right) v(d s)+\sigma^{2} x^{2}$. Since $\psi$ is unbounded the function $W$ is increasing on $(0, \infty)$. Moreover, by [21, Lemma 4]

$$
\frac{1}{2} \psi^{*}(x) \leq W(x) \leq 24 \psi^{*}(x), \quad x \geq 0 .
$$

Since $\psi \in \operatorname{WLSC}(\alpha, \gamma)$ then $\psi^{*} \in \operatorname{WLSC}(\alpha, \gamma)$ and $W \in \operatorname{WLSC}\left(\alpha, \frac{\gamma}{48}\right)$. Now, we can repeat the arguments of [7, Lemma 18] to arrive at Eq. 10.

Lemma 2.14 Let $a>0$. If $\psi \geq a \psi^{*}$, then for $x>0$,

$$
\frac{2}{\pi^{3}} \int_{1 / x}^{\infty} \frac{d s}{\psi^{*}(s)} \leq K(x) \leq \frac{10}{\pi a} \int_{1 / x}^{\infty} \frac{d s}{\psi^{*}(s)} .
$$

Moreover, $\psi \in \operatorname{WLSC}(\alpha, \gamma), \alpha>1$ if and only if

$$
K(x) \approx \frac{1}{|x| \psi(1 / x)} \approx \frac{V^{2}(|x|)}{|x|}, x \neq 0 \text { and } \psi^{*} \approx \psi .
$$

The comparability constants depend only on the scalings. In this case $K \in W L S C\left(\alpha-1, \gamma_{1}\right)$, where $\gamma_{1}>0$ depends on the scalings of $\psi$.

Proof Let $\tilde{K}(x)=\frac{1}{\pi} \int_{0}^{\infty}(1-\cos x s) \frac{1}{\psi^{*}(s)} d s$. Observe that $\tilde{K}$ is the Lévy-Khintchine exponent of some isotropic unimodal Lévy process with the Lévy density $\frac{1}{2 \pi \psi^{*}(|s|)}$. By Lemma 2.12 and the inequality $1-\cos s \leq s^{2} / 2$ we have

$$
\frac{2}{\pi^{2}} \int_{0}^{\infty}\left(1 \wedge(x s)^{2}\right) \frac{d s}{\psi^{*}(s)} \leq \pi \tilde{K}(x) \leq \frac{x^{2}}{2} \int_{0}^{1 / x} s^{2} \frac{d s}{\psi^{*}(s)}+2 \int_{1 / x}^{\infty} \frac{d s}{\psi^{*}(s)} .
$$

Moreover, by Eq. 6, for $0<x s \leq 1$,

$$
(x s / 2)^{2} \psi^{*}(2 / x) \leq 4 \psi^{*}(s),
$$

which implies that

$$
\frac{x^{2}}{2} \int_{0}^{1 / x} s^{2} \frac{d s}{\psi^{*}(s)} \leq \frac{8}{x \psi^{*}(2 / x)} \leq 8 \int_{1 / x}^{2 / x} \frac{d s}{\psi^{*}(s)} \leq 8 \int_{1 / x}^{\infty} \frac{d s}{\psi^{*}(s)} .
$$

Hence,

$$
\frac{2}{\pi^{3}} \int_{1 / x}^{\infty} \frac{d s}{\psi^{*}(s)} \leq \tilde{K}(x) \leq \frac{10}{\pi} \int_{1 / x}^{\infty} \frac{d s}{\psi^{*}(s)} .
$$

Since $\tilde{K}(x) \leq K(x) \leq \frac{1}{a} \tilde{K}(x)$ we get the first conclusion.

Suppose that $\psi \in \operatorname{WLSC}(\alpha, \gamma), \alpha>1$. Then $\psi \geq \gamma \psi^{*}$ and $\psi^{*} \in \operatorname{WLSC}(\alpha, \gamma)$ as well. Hence, for $x>0$,

$$
\frac{1}{16} \frac{1}{x \psi^{*}(1 / x)} \leq \frac{1}{x \psi^{*}(2 / x)} \leq \int_{1 / x}^{\infty} \frac{d r}{\psi^{*}(r)} \leq \frac{1}{\gamma(\alpha-1) x \psi^{*}(1 / x)},
$$

which shows that $K(x) \approx \frac{1}{x \psi(1 / x)}$ with the comparability constant dependent on the scaling characteristics. Also, it is evident that $K$ satisfies the weak lower scaling condition with index $\alpha-1$. Furthermore, by Lemma 2.8 we get $K(x) \approx \frac{V^{2}(|x|)}{|x|}$.

Next we assume that $\frac{1}{\psi^{*}(|x|)} \approx \frac{K(1 / x)}{|x|}$. By [7, Theorem 26] it is equivalent to the fact that $K(x)$ satisfies the global weak lower and upper scaling conditions with indices $0<$ 
$\delta \leq \beta<2$, respectively. This implies that $x K(x)$ satisfies the global weak lower scaling condition with index $\alpha=\delta+1$. Equivalently, $\psi^{*}$ satisfies the weak lower scaling condition with index $\alpha=\delta+1>1$. The proof is completed.

The following technical lemma is the main tool in estimating the tail function of $T_{0}$ via its Laplace transform. Recall that $\tilde{K}(x)=\frac{1}{\pi} \int_{0}^{\infty}(1-\cos x s) \frac{1}{\psi^{*}(s)} d s$.

Lemma 2.15 For any $\lambda>0$,

$$
u^{\lambda}(0) \geq \frac{1}{4} \tilde{K}\left(\frac{1}{\psi^{-1}(\lambda)}\right) \geq \frac{1}{32 \pi^{3}} \frac{\psi^{-1}(\lambda)}{\lambda} .
$$

Let $a>0$. If $a \psi^{*}(x) \leq \psi(x), x \geq 0$, then

$$
\frac{a}{4} K\left(\frac{1}{\psi^{-1}(\lambda)}\right) \leq u^{\lambda}(0) \leq \frac{3 \pi^{2}}{2 a} K\left(\frac{1}{\psi^{-1}(\lambda)}\right) .
$$

For $x \psi^{-1}(\lambda) \leq 1, x \geq 0$,

$$
K^{\lambda}(x) \geq \frac{a}{10 \pi^{2}} K(x) .
$$

Proof By Eq. 6, $\lambda=\psi^{*}\left(\psi^{-1}(\lambda) s s^{-1}\right) \leq\left(2 s^{-2}+2\right) \psi^{*}\left(\psi^{-1}(\lambda) s\right)$, hence

$$
\begin{aligned}
u^{\lambda}(0) & =\frac{1}{\pi} \int_{0}^{\infty} \frac{d r}{\lambda+\psi(r)}=\frac{\psi^{-1}(\lambda)}{\pi} \int_{0}^{\infty} \frac{d s}{\lambda+\psi\left(\psi^{-1}(\lambda) s\right)} \\
& \geq \frac{\psi^{-1}(\lambda)}{\pi} \int_{0}^{\infty} \frac{d s}{\lambda+\psi^{*}\left(\psi^{-1}(\lambda) s\right)} \geq \frac{\psi^{-1}(\lambda)}{\pi}\left[\int_{0}^{1} \frac{d s}{2 \lambda}+\int_{1}^{\infty} \frac{d s}{2 \psi^{*}\left(\psi^{-1}(\lambda) s\right)}\right] \\
& \geq \frac{\psi^{-1}(\lambda)}{2 \pi}\left[\int_{0}^{1} \frac{s^{2} d s}{4 \psi^{*}\left(\psi^{-1}(\lambda) s\right)}+\int_{1}^{\infty}(1-\cos s) \frac{d s}{2 \psi^{*}\left(\psi^{-1}(\lambda) s\right)}\right] \\
& \geq \frac{\psi^{-1}(\lambda)}{4 \pi} \int_{0}^{\infty}(1-\cos s) \frac{d s}{\psi^{*}\left(\psi^{-1}(\lambda) s\right)}=\frac{1}{4} \tilde{K}\left(\frac{1}{\psi^{-1}(\lambda)}\right) .
\end{aligned}
$$

By Eqs. 11 and 12 we have $\tilde{K}(x) \geq \frac{1}{8 \pi^{3}} \frac{1}{x \psi^{*}(1 / x)}$, which implies the second inequality in Eq. 13 .

Now assume that $a \psi^{*}(x) \leq \psi(x), x \geq 0$. Then we have

$$
u^{\lambda}(0) \geq \frac{1}{4} \tilde{K}\left(\frac{1}{\psi^{-1}(\lambda)}\right) \geq \frac{a}{4} K\left(\frac{1}{\psi^{-1}(\lambda)}\right) .
$$

To obtain the upper bound we apply Lemma 2.12 with $f(r)=\frac{1}{\psi^{*}\left(\psi^{-1}(\lambda) r\right)}$ to get

$$
\begin{aligned}
u^{\lambda}(0) & \leq \frac{\psi^{-1}(\lambda)}{\pi}\left[\int_{0}^{1} \frac{d s}{\lambda}+\int_{1}^{\infty} \frac{d s}{\psi\left(\psi^{-1}(\lambda) s\right)}\right] \\
& =\frac{\psi^{-1}(\lambda)}{\pi}\left[3 \int_{0}^{1} \frac{s^{2} d s}{\psi\left(\psi^{-1}(\lambda)\right)}+\int_{1}^{\infty} \frac{d s}{\psi\left(\psi^{-1}(\lambda) s\right)}\right] \\
& \leq \frac{3 \psi^{-1}(\lambda)}{a \pi} \int_{0}^{\infty}\left(s^{2} \wedge 1\right) \frac{d s}{\psi^{*}\left(\psi^{-1}(\lambda) s\right)} \\
& \leq \frac{3 \pi^{2}}{2 a} \frac{\psi^{-1}(\lambda)}{\pi} \int_{0}^{\infty}(1-\cos s) \frac{d s}{\psi^{*}\left(\psi^{-1}(\lambda) s\right)} \\
& \leq \frac{3 \pi^{2}}{2 a} K\left(\frac{1}{\psi^{-1}(\lambda)}\right) .
\end{aligned}
$$


For $0<x \psi^{-1}(\lambda) \leq 1$, applying Lemma 2.12 with $f(s)=\frac{1}{\lambda+\psi^{*}(s)}$, we obtain

$$
\begin{aligned}
\pi K^{\lambda}(x) & =\int_{0}^{\infty}(1-\cos (x s)) \frac{d s}{\lambda+\psi(s)} \geq \int_{0}^{\infty}(1-\cos (x s)) \frac{d s}{\lambda+\psi^{*}(s)} \\
& \geq \frac{2}{\pi^{2}} \int_{0}^{\infty}\left(1 \wedge(x s)^{2}\right) \frac{d s}{\lambda+\psi^{*}(s)} \geq \frac{1}{\pi^{2}} \int_{1 / x}^{\infty} \frac{d s}{\psi^{*}(s)} \\
& \geq \frac{a}{10 \pi} K(x),
\end{aligned}
$$

where the last step follows from Lemma 2.14.

For two functions $g, f$ we write $g(x) \cong f(x), x \rightarrow x_{0}$, if $\lim _{x \rightarrow x_{0}} g(x) / f(x)=1$.

Lemma 2.16 Suppose that $\psi(r)$ is regularly varying at 0 with index $1<\delta \leq 2$. Then

$$
u^{\lambda}(0) \cong \frac{\psi^{-1}(\lambda)}{\lambda} \frac{1}{\delta \sin \frac{\pi}{\delta}}, \quad \lambda \rightarrow 0^{+}
$$

and $u^{\lambda}(0)$ is regularly varying at 0 with index $1 / \delta-1$.

If $\psi(r)$ is regularly varying at 0 with index 1 , then

$$
u^{\lambda}(0) \cong \frac{1}{\pi} \int_{\psi^{-1}(\lambda)}^{\infty} \frac{d s}{\psi(s)}, \quad \lambda \rightarrow 0^{+}
$$

and $u^{\lambda}(0)$ is slowly varying.

Proof Assume that $\psi(s)$ is regularly varying with index $1<\delta \leq 2$. We may write

$$
\begin{aligned}
u^{\lambda}(0) & =\frac{1}{\pi} \int_{0}^{\infty} \frac{d s}{\lambda+\psi(s)}=\frac{1}{\pi} \int_{0}^{1} \frac{d s}{\lambda+\psi(s)}+\frac{1}{\pi} \int_{1}^{\infty} \frac{d s}{\lambda+\psi(s)} \\
& =\frac{\psi^{-1}(\lambda)}{\lambda \pi} \int_{0}^{\frac{1}{\psi^{-1}(\lambda)}} \frac{d w}{1+\frac{\psi\left(\psi^{-1}(\lambda) w\right)}{\psi^{*}\left(\psi^{-1}(\lambda)\right)}}+\frac{1}{\pi} \int_{1}^{\infty} \frac{d s}{\lambda+\psi(s)} .
\end{aligned}
$$

The second integral converges to $\frac{1}{\pi} \int_{1}^{\infty} \frac{d r}{\psi(r)}<\infty$ and since $\frac{\lambda}{\psi^{-1}(\lambda)} \rightarrow 0$ it has no contribution to the limit. Note that $\psi^{*}(u) \cong \psi(u), u \rightarrow 0$ (see [3, Theorem 1.5.3]). Since $\psi(x)>0, x \neq 0$, by Potter's lemma [3, Theorem 1.5.6], and continuity of $\psi$ and $\psi^{*}$, for $1<\delta^{*}<\delta$, we can find a constant $c=c\left(\delta^{*}, \psi\right)>0$ such that for $\lambda<1, \psi^{-1}(\lambda) s<$ $1, s>1$,

By the dominated convergence theorem

$$
\frac{\psi\left(\psi^{-1}(\lambda) s\right)}{\psi^{*}\left(\psi^{-1}(\lambda)\right)} \geq c s^{\delta^{*}}
$$

$$
\lim _{\lambda \rightarrow 0} \frac{\lambda}{\psi^{-1}(\lambda)} u^{\lambda}(0)=\frac{1}{\pi} \int_{0}^{\infty} \frac{d s}{1+s^{\delta}}=\frac{\Gamma(1 / \delta) \Gamma(1-1 / \delta)}{\pi \delta}=\frac{1}{\delta \sin \frac{\pi}{\delta}} .
$$

Next, let $\psi(r)$ be regularly varying at 0 with index 1 . Let $L(u)=\int_{u}^{\infty} \frac{d r}{\psi(r)}$. This function is slowly varying at $0\left[3\right.$, Proposition 1.5.9a]. Note that $\int_{0}^{\psi^{-1}(\lambda)} \frac{d r}{\lambda+\psi(r)} \leq \frac{\psi^{-1}(\lambda)}{\lambda}=$ $\frac{\psi^{-1}(\lambda)}{\psi^{*}\left(\psi^{-1}(\lambda)\right)}$. Due to regular variation of $\psi^{2}$ with index 2 we have

$$
\int_{\psi^{-1}(\lambda)}^{\infty} \frac{d r}{\psi(r)}-\int_{\psi^{-1}(\lambda)}^{\infty} \frac{d r}{\lambda+\psi(r)} \leq \lambda \int_{\psi^{-1}(\lambda)}^{\infty} \frac{d r}{\psi^{2}(r)} \cong \frac{\psi^{-1}(\lambda)}{\lambda}, \quad \lambda \rightarrow 0^{+} .
$$


Hence it is enough to prove that

$$
\frac{u / \psi^{*}(u)}{L(u)} \rightarrow 0, \quad u \rightarrow 0^{+}
$$

Let $a>1$. Then

$$
\frac{L(u)-L(a u)}{L(u)} \geq \frac{(a-1) u}{L(u) \psi^{*}(a u)} .
$$

Since $L$ varies slowly the left hand side converges to 0 so the proof is completed.

\section{Hitting Times of Points}

In this section we examine the tail function $\mathbb{P}^{x}\left(T_{0}>t\right)$ under various assumptions on $\psi$ or $K$. Under the monotonicity of $K$ we find the lower and upper bounds of the tail function. On the other hand comparability of $\psi$ and $\psi^{*}$ is another source of the estimates via approximate inversion of the Laplace transform. We also derive asymptotics of $\mathbb{P}^{x}\left(T_{0}>t\right)$ as $t \rightarrow \infty$ by applying Tauberian theorems.

Proposition 3.1 We have for any $t>0$ and $x \in \mathbb{R}$

$$
\mathbb{P}^{x}\left(T_{0}>t\right) \leq\left[7 \frac{K(x)}{\tilde{K}\left(\frac{1}{\psi^{-1}(1 / t)}\right)}\right] \wedge 1 \leq\left[51 \pi^{3} \frac{K(x)}{t \psi^{-1}(1 / t)}\right] \wedge 1 .
$$

Let $a>0$. If additionally $\psi(x) \geq a \psi^{*}(|x|)$ for $x \in \mathbb{R}$, then

$$
\mathbb{P}^{x}\left(T_{0}>t\right) \leq\left[\frac{7}{a} \frac{K(x)}{K\left(\frac{1}{\psi^{-1}(1 / t)}\right)}\right] \wedge 1 .
$$

Proof Observe that for the Laplace transform of $\mathbb{P}^{x}\left(T_{0}>\cdot\right)$ we have

$$
\mathcal{L}\left(\mathbb{P}^{x}\left(T_{0}>\cdot\right)\right)(\lambda)=\frac{1}{\lambda}\left[1-\mathbb{E}^{x} e^{-\lambda T_{0}}\right]=\frac{1}{\lambda} \frac{u^{\lambda}(0)-u^{\lambda}(x)}{u^{\lambda}(0)} \leq \frac{1}{\lambda} \frac{K(x)}{u^{\lambda}(0)} .
$$

It follows from Eq. 13 that

$$
\mathcal{L}\left(\mathbb{P}^{x}\left(T_{0}>\cdot\right)\right)(\lambda) \leq \frac{4}{\lambda} \frac{K(x)}{\tilde{K}\left(\frac{1}{\psi^{-1}(\lambda)}\right)} \leq 32 \pi^{3} \frac{K(x)}{\psi^{-1}(\lambda)}
$$

in general case, while under the assumption $\psi(x) \geq a \psi^{*}(|x|)$,

By [7, Lemma 5] we have

$$
\mathcal{L}\left(\mathbb{P}^{x}\left(T_{0}>\cdot\right)\right)(\lambda) \leq \frac{4}{a \lambda} \frac{K(x)}{K\left(\frac{1}{\psi^{-1}(\lambda)}\right)} .
$$

$$
\mathbb{P}^{x}\left(T_{0}>t\right) \leq \frac{4 e}{e-1} \frac{K(x)}{\tilde{K}\left(\frac{1}{\psi^{-1}(1 / t)}\right)} \leq 32 \pi^{3} \frac{e}{e-1} \frac{K(x)}{t \psi^{-1}(1 / t)}
$$

in general case, and in the other considered case

$$
\mathbb{P}^{x}\left(T_{0}>t\right) \leq \frac{e}{e-1} \frac{4}{a} \frac{K(x)}{K\left(\frac{1}{\psi^{-1}(1 / t)}\right)},
$$

which ends the proof. 
Proposition 3.2 Let $K$ be non-decreasing on $[0, \infty)$ and $\kappa=0$. Then

$$
\mathbb{P}^{x}\left(T_{0}>t\right) \leq\left[8 \frac{K(x)}{K\left(R_{t}\right)}\right] \wedge 1
$$

where $R_{t} K\left(R_{t}\right)=t$.

Proof Let $R, t>0$. We have

$$
\mathbb{P}^{x}\left(T_{0}>t\right) \leq \mathbb{P}^{x}\left(\tau_{(-R, R)} \wedge T_{0}>t\right)+\mathbb{P}^{x}\left(\tau_{(-R, R)}<T_{0}\right) .
$$

Let $|x|<R$. By Chebyshev's inequality and Proposition 2.6 we obtain

$$
\mathbb{P}^{x}\left(\tau_{(-R, R)} \wedge T_{0}>t\right) \leq \frac{\mathbb{E}^{x} \tau_{(-R, R)} \wedge T_{0}}{t} \leq 4 \frac{R K(x)}{t},
$$

while by Lemma 2.7 ,

$$
\mathbb{P}^{x}\left(\tau_{(-R, R)}<T_{0}\right) \leq 4 \frac{K(x)}{K(R)} .
$$

Setting $R K(R)=t$ we obtain the conclusion.

Lemma 3.3 Let $K$ be non-decreasing on $[0, \infty)$. For $x \in \mathbb{R}, t>0$,

$$
\mathbb{P}^{x}\left(T_{0}>t\right) \geq \frac{C_{3}}{6 C_{1}}\left(\frac{K(x)}{K\left(1 / \psi^{-1}(1 / t)\right)} \wedge 1\right) .
$$

Proof For $x \geq 1 / \psi^{-1}(1 / t)$ we have, by Lemma $2.8, V(x) \geq V\left(1 / \psi^{-1}(1 / t)\right) \geq \frac{\sqrt{t}}{C_{1}}$. Hence by Lemma 2.10,

$$
\mathbb{P}^{x}\left(T_{0}>t\right) \geq \mathbb{P}^{x}\left(\tau_{(0, \infty)}>t\right) \geq \frac{C_{3}}{C_{1}} .
$$

Let $R=1 / \psi^{-1}(1 / t)$. Then for $0<x<R$, by Proposition 2.7 and the strong Markov property

$$
\begin{aligned}
\mathbb{P}^{x}\left(T_{0}>t\right) & \geq \mathbb{E}^{x}\left\{\left|X_{\tau_{(-R, R)} \wedge T_{0}}\right| \geq R ; \mathbb{P}^{\left.X_{\tau_{(-R, R)} \wedge T_{0}}\left(T_{0}>t\right)\right\}}\right. \\
& \geq \frac{C_{3}}{C_{1}} \mathbb{P}^{x}\left\{\left|X_{\tau_{(-R, R)} \wedge T_{0}}\right| \geq R\right\} \geq \frac{C_{3}}{6 C_{1}} \frac{K(x)}{K(R)} .
\end{aligned}
$$

The proof is completed.

The assumption about monotonicity of $K$ can be removed if we assume the lower scaling condition of $\psi$.

Proposition 3.4 Let $\psi \in W L S C(\alpha, \gamma), \alpha>1$. For $x \in \mathbb{R}, t>0$,

$$
\mathbb{P}^{x}\left(T_{0}>t\right) \geq c\left(\frac{K(x)}{K\left(1 / \psi^{-1}(1 / t)\right)} \wedge 1\right),
$$

where $c$ depends only on the scalings.

Proof Since $\psi \in \operatorname{WLSC}(\alpha, \gamma)$ we have $\gamma \psi^{*}(r) \leq \psi(r)$ for any $r \geq 0$. By Lemma 2.15,

$$
\frac{2 \gamma}{3 \pi^{2}} \frac{K^{\lambda}(x)}{K\left(\frac{1}{\psi^{-1}(\lambda)}\right)} \leq \lambda \mathcal{L}\left(\mathbb{P}^{x}\left(T_{0}>\cdot\right)\right)(\lambda) \leq \frac{4}{\gamma} \frac{K^{\lambda}(x)}{K\left(\frac{1}{\psi^{-1}(\lambda)}\right)} .
$$


This, Lemma 2.14 and Eq. 10 imply for $\lambda>0$ and $s>1$,

$$
\frac{\mathcal{L}\left(\mathbb{P}^{x}\left(T_{0}>\cdot\right)\right)(\lambda s)}{\mathcal{L}\left(\mathbb{P}^{x}\left(T_{0}>\cdot\right)\right)(\lambda)} \leq \frac{6 \pi^{2}}{\gamma^{2}} \frac{1}{s} \frac{K^{\lambda s}(x)}{K^{\lambda}(x)} \frac{K\left(\frac{1}{\psi^{-1}(\lambda)}\right)}{K\left(\frac{1}{\psi^{-1}(\lambda s)}\right)} \leq c_{1} \frac{\psi^{-1}(\lambda)}{\psi^{-1}(\lambda s)} \leq c_{2} s^{-1 / 2},
$$

where $c_{2}$ depends only on the scalings. Hence, by [7, Lemma 13] there exists a constant $c_{3}$ that depends only on the scalings such that

$$
\mathbb{P}^{x}\left(T_{0}>t\right) \geq c_{3} \frac{K^{1 / t}(x)}{K\left(\frac{1}{\psi^{-1}(1 / t)}\right)} .
$$

For $t \geq 1 / \psi^{*}(1 / x)$, by Lemma $2.15, K^{1 / t}(x) \geq \frac{\gamma}{10 \pi^{2}} K(x)$, which gives the conclusion in this case. We complete the proof by applying (14) for $t<1 / \psi^{*}(1 / x)$.

From the above lower and upper bounds we derive two corollaries providing two sided sharp estimates.

Corollary 3.5 If $X$ is unimodal then for $x \in \mathbb{R}$ and $t>0$,

$$
\mathbb{P}^{x}\left(T_{0}>t\right) \approx \frac{K(x)}{K\left(1 / \psi^{-1}(1 / t)\right)} \wedge 1 .
$$

The comparability constant is absolute.

Proof If $X$ is unimodal then $K$ is increasing and $\psi \geq \pi^{-2} \psi^{*}$ (see [7, Proposition 2]), hence the upper bound follows from Proposition 3.1, while the lower bound is a consequence of Lemma 3.3.

Corollary 3.6 Let $\psi \in W L S C(\alpha, \gamma), \alpha>1$. Then for $x \in \mathbb{R}$ and $t>0$,

$$
\mathbb{P}^{x}\left(T_{0}>t\right) \approx \frac{K(x)}{K\left(1 / \psi^{-1}(1 / t)\right)} \wedge 1 \approx \frac{1}{t \psi^{-1}(1 / t)|x| \psi(1 / x)} \wedge 1 .
$$

The comparability constants depend on the scalings.

Proof By Lemma 2.14 we have $K(x) \approx \frac{1}{|x| \psi(1 / x)}$ and the conclusion follows immediately from Proposition 3.1 and Proposition 3.4.

Example 1 Let $\psi(x)=|x|+x^{2}$. The corresponding process, which is the sum of the Cauchy process and independent Brownian motion, is obviously unimodal. Then, by Lemma 2.14,

$$
K(x) \approx \int_{1 / x}^{\infty} \frac{d r}{\psi(r)} \approx \log (1+x)
$$

and by Corollary 3.5,

$$
\mathbb{P}^{x}\left(T_{0}>t\right) \approx \frac{\log (1+x)}{\log (1+\sqrt{t})} \wedge 1
$$

Example 2 We consider $\psi(x)=2 \int_{0}^{1}(1-\cos (|x| s)) v(d s)$, where $v$ is singular. Namely, let $v(d s)=\sum_{k=1}^{\infty} \delta_{1 / k}(d s)\left(k^{\alpha}-(k-1)^{\alpha}\right)$ or $v(d s)=s^{-\beta} F(d s)$, where $\alpha \in(1,2)$ and $\beta=\alpha+\log 2 / \log 3$ and $F$ is the standard Cantor measure on [0,1]. In both cases we claim that $\psi(x) \approx|x|^{\alpha} \wedge x^{2}$. Indeed, by the integration by parts and [20, Lemma 2] to 
verify the claim in the second case, we obtain that $\int_{0}^{|x|^{-1}} s^{2} v(d s) \approx 1 \wedge|x|^{\alpha-2}$. Moreover $\int_{|x|^{-1}}^{\infty} v(d s) \leq c|x|^{\alpha}$ for $|x| \geq 1$ and $\int_{|x|^{-1}}^{\infty} v(d s)=0$ for $|x|<1$. Since

$$
2(1-\cos 1)|x|^{2} \int_{0}^{|x|^{-1}} s^{2} v(d s) \leq \psi(x) \leq|x|^{2} \int_{0}^{|x|^{-1}} s^{2} v(d s)+2 \int_{|x|^{-1}}^{\infty} v(d s)
$$

we get the claim in both cases. Hence $\psi \in \operatorname{WLSC}(\alpha, \gamma)$ for some $0<\gamma \leq 1$. Applying Corollary 3.6 we obtain

$$
\mathbb{P}^{x}\left(T_{0}>t\right) \approx \frac{|x|^{\alpha-1} \vee|x|}{t^{1-1 / \alpha} \vee t^{1 / 2}} \wedge 1
$$

Since the Lévy measure is singular the process with symbol $\psi(x)$ can not be unimodal. This illustrates that Corollary 3.6 does not follow from Corollary 3.5.

Remark 1 There are recent results obtained by Juszczyszyn and Kwaśnicki [25] where not only the behaviour of the tail function $\mathbb{P}^{x}\left(T_{0}>t\right)$ was described but also its derivatives. Their assumptions on the process were much more restrictive than ours. They assumed complete monotonicity of the Lévy density and some additional property of the first two derivatives of the symbol of the process. The results of our paper regarding the tail functions are more general, however our methods do not allow us to treat the derivatives. The processes from Example 1 and 2 do not satisfy the assumptions of [25]. In Example 1 the symbol $\psi$ fails the requirements of [25], while the Lévy measures in Example 2 are singular.

Now we turn to asymptotics of the tail function when $t \rightarrow \infty$ not only in the case of hitting $\{0\}$ but for hitting arbitrary compact set as well.

Proposition 3.7 Let $\psi$ be regularly varying at 0 with index $\delta \in(1,2]$. Then for a compact set $B$ such that $0 \in B$ we have for $x \in \mathbb{R}$,

$$
\lim _{t \rightarrow \infty} t \psi^{-1}(1 / t) \mathbb{P}^{x}\left(T_{B}>t\right)=\frac{\delta \Gamma\left(1-\frac{1}{\delta}\right) \sin ^{2} \frac{\pi}{\delta}}{\pi}\left[K(x)-\mathbb{E}^{x} K\left(X_{T_{B}}\right)\right] .
$$

If $\psi$ is regularly varying at 0 with index 1 then there is a function $L(u)$ slowly varying at 0 such that

$$
\lim _{t \rightarrow \infty} L(1 / t) \mathbb{P}^{x}\left(T_{B}>t\right)=K(x)-\mathbb{E}^{x} K\left(X_{T_{B}}\right) .
$$

We can take $L(u)=\frac{1}{\pi} \int_{\psi^{-1}(u)}^{\infty} \frac{d r}{\psi(r)}, u>0$.

Proof Observe that

$$
\mathcal{L}\left(\mathbb{P}^{x}\left(T_{B}>\cdot\right)\right)(\lambda)=\frac{1}{\lambda}\left[1-\mathbb{E}^{x} e^{-\lambda T_{B}}\right] .
$$

Since $0 \in B, 0$ is regular for $B$. By symmetry $G_{B^{c}}^{\lambda}(x, 0)=0$. Hence

$$
\begin{aligned}
\lambda u^{\lambda}(0) \mathcal{L}\left(\mathbb{P}^{x}\left(T_{B}>\cdot\right)\right)(\lambda)= & u^{\lambda}(0)-u^{\lambda}(x)-\mathbb{E}^{x} e^{-\lambda T_{B}}\left[u^{\lambda}(0)-u^{\lambda}\left(X_{T_{B}}\right)\right] \\
& +u^{\lambda}(x)-\mathbb{E}^{x} e^{-\lambda T_{B}}\left(u^{\lambda}\left(X_{T_{B}}\right)\right) \\
= & u^{\lambda}(0)-u^{\lambda}(x)-\mathbb{E}^{x} e^{-\lambda T_{B}}\left[u^{\lambda}(0)-u^{\lambda}\left(X_{T_{B}}\right)\right]+G_{B^{c}}^{\lambda}(x, 0) \\
= & K^{\lambda}(x)-\mathbb{E}^{x} e^{-\lambda T_{B}} K^{\lambda}\left(X_{T_{B}}\right) .
\end{aligned}
$$

Since $K$ is continuous and $B$ is compact, by the dominated convergence theorem and Lemma 2.1,

$$
\lim _{\lambda \rightarrow 0} \lambda u^{\lambda}(0) \mathcal{L}\left(\mathbb{P}^{x}\left(T_{B}>\cdot\right)\right)(\lambda)=K(x)-\mathbb{E}^{x} K\left(X_{T_{B}}\right) .
$$


Let $\psi$ be regularly varying at 0 with index $\delta \in(1,2]$. By Lemma 2.16 ,

$$
\lim _{\lambda \rightarrow 0} \psi^{-1}(\lambda) \mathcal{L}\left(\mathbb{P}^{x}\left(T_{B}>\cdot\right)\right)(\lambda)=\delta \sin \left(\frac{\pi}{\delta}\right)\left[K(x)-\mathbb{E}^{x} K\left(X_{T_{B}}\right)\right] .
$$

Define $U(s)=\int_{0}^{s} \mathbb{P}^{x}\left(T_{B}>t\right) d t$. We have

$$
\mathcal{L} U(\lambda)=\frac{1}{\lambda} \mathcal{L}\left(\mathbb{P}^{x}\left(T_{B}>\cdot\right)\right)(\lambda)
$$

Hence

$$
\lim _{\lambda \rightarrow 0} \lambda \psi^{-1}(\lambda) \mathcal{L} U(\lambda)=\delta \sin \left(\frac{\pi}{\delta}\right)\left[K(x)-\mathbb{E}^{x} K\left(X_{T_{B}}\right)\right] .
$$

Since $\psi^{-1}$ is regularly varying at 0 with index $1 / \delta$ the Tauberian theorem ([3, Theorem 1.7.1]) implies

$$
\lim _{t \rightarrow \infty} \psi^{-1}(1 / t) U(t)=\frac{\delta \sin \left(\frac{\pi}{\delta}\right)}{\Gamma(1+1 / \delta)}\left[K(x)-\mathbb{E}^{x} K\left(X_{T_{B}}\right)\right] .
$$

By the monotone density theorem ([3, Theorem 1.7.2]),

$$
\lim _{t \rightarrow \infty}\left[t \psi^{-1}(1 / t) \mathbb{P}^{x}\left(T_{B}>t\right)\right]=\frac{\delta \sin \frac{\pi}{\delta}}{\Gamma(1 / \delta)}\left[K(x)-\mathbb{E}^{x} K\left(X_{T_{B}}\right)\right] .
$$

If $\psi$ is regularly varying at 0 with index 1 , then by Lemma 2.16 ,

$$
u^{\lambda}(0) \cong \frac{1}{\pi} \int_{\psi^{-1}(\lambda)}^{\infty} \frac{d r}{\psi(r)}=L(\lambda)
$$

where $L(\lambda)$ is slowly varying at 0 . Hence

$$
\lim _{\lambda \rightarrow 0} \lambda L(\lambda) \mathcal{L}\left(\mathbb{P}^{x}\left(T_{B}>\cdot\right)\right)(\lambda)=K(x)-\mathbb{E}^{x} K\left(X_{T_{B}}\right) .
$$

By the monotone density theorem

$$
\lim _{t \rightarrow \infty} L(1 / t) \mathbb{P}^{x}\left(T_{B}>t\right)=K(x)-\mathbb{E}^{x} K\left(X_{T_{B}}\right) .
$$

Corollary 3.8 Let $\psi$ be regularly varying at 0 with index $\delta \in[1,2]$. Then for $x \in \mathbb{R}$,

$$
\lim _{t \rightarrow \infty}\left[t \psi^{-1}(1 / t) \mathbb{P}^{x}\left(T_{0}>t\right)\right]=\frac{\delta \Gamma\left(1-\frac{1}{\delta}\right) \sin ^{2} \frac{\pi}{\delta}}{\pi} K(x), \quad \delta>1,
$$

and

where $L(u)=\frac{1}{\pi} \int_{\psi^{-1}(u)}^{\infty} \frac{d r}{\psi(r)}$.

$$
\lim _{t \rightarrow \infty} L(1 / t) \mathbb{P}^{x}\left(T_{0}>t\right)=K(x), \quad \delta=1,
$$

Proof Since $\mathbb{E}^{x} K\left(X_{T_{0}}\right)=0$ the corollary follows from Proposition 3.7.

Remark 2 We again compare [25] with our results with regard to asymptotics of the tail function of $T_{0}$. For example the case $\psi(x)=|x|+|x|^{2}$ is not covered in [25]. In Example 2 we provided sharp estimates of $\mathbb{P}^{x}\left(T_{0}>t\right)$ and Corollary 3.8 exhibits the asymptotics at infinity. Note that $\psi^{-1}(u)=\frac{u}{\sqrt{u+\frac{1}{4}}+\frac{1}{2}} \cong u, \quad u \rightarrow 0$. Next,

$L(u)=\frac{1}{\pi} \int_{\psi^{-1}(u)}^{\infty} \frac{d r}{r+r^{2}}=\frac{1}{\pi}\left[\log \left(u+\sqrt{u+\frac{1}{4}}+\frac{1}{2}\right)-\log u\right] \cong-\frac{\log u}{\pi}, \quad u \rightarrow 0^{+}$. 
Then, by the second part of Corollary 3.8,

$$
\lim _{t \rightarrow \infty} \log t \mathbb{P}^{x}\left(T_{0}>t\right)=\pi K(x),
$$

where $K(x) \approx \log (1+x)$.

The next example illustrates that the decay of the tail function of $T_{0}$ can be very slow. Note that the intensity of small jumps of the process below is larger than the corresponding intensity of the Cauchy process while it is smaller than the corresponding intensity for any symmetric $\alpha$-stable process, $\alpha>1$. Therefore the considered process is in some sense between the Cauchy process and any symmetric $\alpha$-stable processes, $\alpha>1$. Note that the Cauchy process hits points with 0 probability, while $\alpha$-stable processes, $\alpha>1$, hit points with probability 1 .

Example 3 Let $v(r)=\frac{\log ^{2}(2+1 /|r|) \log (2+|r|)}{r^{2}}, r \in \mathbb{R}$. By Lemma 2.12,

$$
\psi(x) \approx \int_{0}^{\infty}\left(1 \wedge(x r)^{2}\right) v(r) d r=x^{2} \int_{0}^{1 / x} r^{2} v(r) d r+\int_{1 / x}^{\infty} v(r) d r .
$$

Elementary calculations show that

$$
\psi(x) \approx x \log ^{2}(2+x) \log (2+1 / x), \quad x \geq 0
$$

and

$$
\psi^{-1}(x) \approx \frac{x}{\log ^{2}(2+x) \log (2+1 / x)}, \quad x \geq 0 .
$$

It is clear that $\psi$ can not have the weak lower scaling property with any $1<\alpha \leq 2$, but $\psi=\psi^{*}$ since $v(r) / r$ is decreasing on $(0, \infty)$. Hence, by Lemma 2.14,

$$
K(x) \approx \int_{1 / x}^{\infty} \frac{d r}{\psi(r)} \approx \frac{\log \log (2+x)}{\log (2+1 / x)}, \quad x>0 .
$$

Moreover, $\psi(x) \cong c x \log (1 / x)$ and $\psi^{-1}(x) \cong \frac{x}{c \log (1 / x)}$ as $x \rightarrow 0^{+}$, where $c=$ $2 \log ^{2} 2 \int_{0}^{\infty} \frac{1-\cos r}{r^{2}} d r=\pi \log ^{2} 2$. Therefore $\psi$ is regularly varying at 0 with index 1 . Hence

$$
L(u)=\frac{1}{\pi} \int_{\psi^{-1}(u)}^{\infty} \frac{d r}{\psi(r)} \cong \frac{1}{c \pi} \log \log (1 / u), \quad u \rightarrow 0^{+}
$$

and from Corollary 3.8 we infer that

$$
\mathbb{P}^{x}\left(T_{0}>t\right) \cong(\pi \log 2)^{2} \frac{K(x)}{\log \log t}, \quad t \rightarrow \infty .
$$

\section{Behaviour of Harmonic Functions}

This section prepares some tools used in the sequel for estimating the tail function for the hitting time of an interval. On the other hand the results are interesting on their own. In the first subsection we prove the global Harnack inequality under global weak scaling assumption for $\psi$, while in the second we provide some boundary type estimates for harmonic functions.

We start with a lemma which shows a very useful property of the compensated kernel. 
Lemma 4.1 Let $0<R \leq \infty$. For $x>0$ we have $\mathbb{E}^{x} K\left(X_{\tau_{(0, R)}}\right) \leq K(x)$.

Proof We provide the proof for $R=\infty$, since the case $R<\infty$ is similar. By [41, Theorem 1.1] we know that the function $K(x)$ is invariant for the killed process upon hitting $\{0\}$ which implies its harmonicity on $\{0\}^{c}$. Let $R>1$ such that $1 / R<x<R$. Then by harmonicity $\mathbb{E}^{x} K\left(X_{\tau_{(1 / R, R)}}\right)=K(x)$. Since $\tau_{(1 / R, R)} \uparrow \tau_{(0, \infty)}, R \uparrow \infty$ and $\tau_{(0, \infty)}<\infty$ almost surely, the conclusion follows by continuity of $K$, quasi-left continuity of $X$ and Fatou's lemma.

\subsection{Harnack inequality}

We say that the global Harnack inequality holds if there is a constant $C_{H}$ such that for every $R>0$ and any non-negative harmonic function on $(-R, R)$ we have

$$
\sup _{x \in(-R / 2, R / 2)} h(x) \leq C_{H} \inf _{x \in(-R / 2, R / 2)} h(x) .
$$

Here we prove that $\psi \in \operatorname{WLSC}(\alpha, \gamma), \alpha>1$ is a sufficient condition. The Harnack inequality will be very important in the next subsection to find the boundary behaviour of certain harmonic functions.

Lemma 4.2 Let $\psi \in W L S C(\alpha, \gamma), \alpha>1$. Then there are $\lambda_{1}, \lambda_{2}$ depending on the scalings such that for any $R>0$,

$$
G_{(-R, R)}(x, y) \geq \lambda_{2} K(R), \quad|x|,|y| \leq \lambda_{1} R .
$$

Proof Since $\psi \in \operatorname{WLSC}(\alpha, \gamma), \alpha>1$, then $K$ has the lower scaling property with index $\alpha-1$. Hence there exists $\delta<1 / 2$, depending on the scalings, such that

$$
\inf _{z \geq R(1-\delta)} K(z)-\sup _{|z| \leq \delta R} K(z) \geq(1 / 2) \inf _{z \geq R(1-\delta)} K(z) \geq(1 / 2) \inf _{z \geq R / 2} K(z) .
$$

Let $\lambda=\frac{\delta}{2}$ and $|x|,|y| \leq \lambda R$. By Lemma 4.1 we have $\mathbb{E}^{x+R} K\left(X_{\tau_{(0,2 R)}}\right) \leq K(x+R)$. Hence

$$
\begin{aligned}
G_{(-R, R)}(x, y) & =G_{(0,2 R)}(x+R, y+R)=G_{\{0\}^{c}}(x+R, y+R)-\mathbb{E}^{x+R} G_{\{0\}^{c}}\left(X_{\tau_{(0,2 R)}}, y+R\right) \\
& =K(x+R)-K(y-x)-\mathbb{E}^{x+R} K\left(X_{\tau_{(0,2 R)}}\right)+\mathbb{E}^{x+R} K\left(X_{\tau_{(0,2 R)}}-y-R\right) \\
& \geq \mathbb{E}^{x+R} K\left(X_{\tau_{(0,2 R)}}-y-R\right)-K(y-x) \\
& \geq \inf _{z \geq R(1-\lambda)} K(z)-\sup _{|z| \leq 2 \lambda R} K(z) \\
& \geq(1 / 2) \inf _{z \geq R / 2} K(z) \geq \lambda_{2} K(R),
\end{aligned}
$$

where $\lambda_{2}$ depends only on the scalings.

Proposition 4.3 Let $\psi \in \operatorname{WLSC}(\alpha, \gamma), \alpha>1$ and let $\lambda_{1}$ be the constant from Lemma 4.2. There exists a constant $\delta \leq \lambda_{1}$ dependent only on the scalings such that for any $R>0$ and any non-empty Borel $A \subset(-\delta R, \delta R)$,

$$
\mathbb{P}^{x}\left(T_{A}<\tau_{(-R, R)}\right) \geq \frac{1}{2}, \quad|x| \leq \delta R .
$$

Proof Let $|a| \leq R / 4$. Let $D=(-R / 2,0) \cup(0, R / 2)$. By Eq. 9 and then by Proposition 2.6, for $|x-a| \leq R / 4$,

$$
\mathbb{P}^{x}\left(T_{a}>\tau_{(-R, R)}\right) \leq \mathbb{P}^{x-a}\left(T_{0}>\tau_{(-R / 2, R / 2)}\right) \leq C_{2} \frac{\mathbb{E}^{x-a} \tau_{D}}{V^{2}(R / 2)} \leq 8 C_{2} K(x-a) \frac{R}{V^{2}(R)} .
$$


Since $\frac{V^{2}(R)}{R} \approx K(R)$, with comparability constant dependent on the scalings, then

$$
\mathbb{P}^{x}\left(T_{a}>\tau_{(-R, R)}\right) \leq c \frac{K(x-a)}{K(R)}, \quad|x-a|<R / 4,
$$

with $c$ dependent on the scalings. Next, we can use WLSC property for $K$ with index $\alpha-1$ to choose $\delta<1 / 2$ (dependent only on the scalings) small enough, such that

$$
\mathbb{P}^{x}\left(T_{a}>\tau_{(-R, R)}\right) \leq 1 / 2, \quad|x-a|<2 \delta R .
$$

Let $x \in A \subset(-\delta R, \delta R)$ and $a \in A$. Then

$$
\mathbb{P}^{x}\left(T_{A}>\tau_{(-R, R)}\right) \leq \mathbb{P}^{x}\left(T_{a}>\tau_{(-R, R)}\right) \leq 1 / 2
$$

Let $R_{0}=\delta R$, with $\delta$ chosen in the preceding proposition.

Proposition 4.4 Let $\psi \in W L S C(\alpha, \gamma), \alpha>1$. Then for any $R>0$, and any non-negative function $F$ such that $(\operatorname{supp} F)^{c} \subset(-R, R)$,

$$
\mathbb{E}^{x} F\left(X_{\tau_{\left(-R_{0}, R_{0}\right)}}\right) \leq \frac{2}{\lambda_{2}} \mathbb{E}^{y} F\left(X_{\tau_{(-R, R)}}\right), \quad|x|,|y|<R_{0} .
$$

Proof Denote $v(w, A)=v(A-w), w \in \mathbb{R}$ and Borel $A \subset \mathbb{R}$. By the Ikeda-Watanabe formula and Lemma 4.2,

$$
\begin{aligned}
\mathbb{E}^{y} F\left(X_{\tau_{(-R, R)}}\right) & \geq \int_{(-R, R)^{c}} \int_{-R_{0}}^{R_{0}} F(z) G_{(-R, R)}(y, w) v(w, d z) d w \\
& \geq \lambda_{2} K(R) \int_{(-R, R)^{c}} \int_{-R_{0}}^{R_{0}} F(z) v(w, d z) d w .
\end{aligned}
$$

Again, by the Ikeda-Watanabe formula, subadditivity of $K$ and Proposition 2.4,

$$
\begin{aligned}
\mathbb{E}^{x} F\left(X_{\left.\tau_{\left(-R_{0}, R_{0}\right)}\right)}\right. & \leq \int_{(-R, R)^{c}} \int_{-R_{0}}^{R_{0}} F(z) G_{\{0\}^{c}}\left(x+R_{0}, w+R_{0}\right) v(w, d z) d w \\
& \leq 2 K\left(R_{0}\right) \int_{(-R, R)^{c}} \int_{-R_{0}}^{R_{0}} F(z) v(w, d z) d w .
\end{aligned}
$$

Hence

$$
\mathbb{E}^{x} F\left(X_{\tau_{\left(-R_{0}, R_{0}\right)}}\right) \leq \frac{2}{\lambda_{2}} \mathbb{E}^{y} F\left(X_{\tau_{(-R, R)}}\right) .
$$

Theorem 4.5 If $\psi \in W L S C(\alpha, \gamma), \alpha>1$, then the global scale invariant Harnack inequality holds. That is there is a constant $C_{H}$ dependent only the scalings such that for any $R>0$ and any non-negative harmonic function on $(-R, R)$ we have

$$
\sup _{x \in(-R / 2, R / 2)} h(x) \leq C_{H} \inf _{x \in(-R / 2, R / 2)} h(x) .
$$

Proof We prove the result for bounded harmonic functions. The boundedness assumption can be removed in a similar way as in [38, Theorem 2.4]. 
With Propositions 4.4 and 4.3 at hand we can use the approach of Bass and Levin ([1]) to get the existence of constants $c_{1}=c_{1}(\alpha, \gamma)$ and $a=a(\alpha, \gamma)<1$ such that, for any function $h$ non-negative and bounded on $\mathbb{R}$ and harmonic in a ball $(-R, R), R>0$,

$$
\sup _{x \in(-a R, a R)} h(x) \leq c_{1} \inf _{x \in(-a R, a R)} h(x) .
$$

Next, we use the standard chain argument to get

$$
\sup _{x \in(-R / 2, R / 2)} h(x) \leq C_{H} \inf _{x \in(-R / 2, R / 2)} h(x),
$$

where $C_{H}=C_{H}\left(c_{1}, a\right)$.

\subsection{Boundary behaviour}

In this subsection we prove certain estimates of non-negative functions which are harmonic on $(0, R), 0<R \leq \infty$. We show that under appropriate assumptions the function $V(x)$ provides the right order of decay at the boundary at 0 for harmonic functions we consider. The obtained results are then used in Section 5 to estimate the tail function of the hitting time of an interval.

In our development the following Property $(\mathbf{H})$ of the derivative of $V$ is crucial. Recall that $V$ is the renewal function defined on page 8. Below, in Remark 3, we discus the situations when it holds. We also mention that we do not know any example of a symmetric Lévy process with an unbounded symbol for which the property is not satisfied.

Property $(\mathbf{H})$.

We say that $X$ satisfies $(\mathbf{H})$ if there is a constant $H \geq 1$ such that for any $0<\delta \leq w \leq$ $u \leq w+2 \delta$ we have

$$
V^{\prime}(u) \leq H V^{\prime}(w)
$$

Remark 3 The assumption $(\mathbf{H})$ is satisfied in the following situations:

a) $\psi \in \operatorname{WLSC}(\alpha, \gamma), \alpha>1$. The constant $H$ depends only on the scalings. Since $V^{\prime}$ is harmonic on $(0, \infty)$ this follows from Theorem 4.5 .

b) $X$ is a subordinate Brownian motion and $\psi \in \operatorname{WLSC}(\beta, \gamma), \beta>0$. The constant $H$ depends only on the scalings. This follows from [21, Theorem 7].

c) $X$ is a special subordinate Brownian motion, since in this case $V^{\prime}$ is non-increasing [5, Lemma 7.5]. Recall that $X$ is a special subordinate Brownian motion if its symbol $\psi(x)=\phi\left(x^{2}\right), x \in \mathbb{R}$, where $\phi$ is a special Bernstein function. That is both $\phi(\lambda), \lambda / \phi(\lambda), \lambda>0$, are Bernstein functions.

Lemma 4.6 Suppose that $(\boldsymbol{H})$ holds. Then for $0<x<\delta<y / 3$ we have

$$
G_{(0, \infty)}(x, y) \leq H^{2} G_{(0, \infty)}(2 \delta, y) \frac{V(x)}{V(\delta)} .
$$

Proof Let $0 \leq u \leq x$. Since $x<y$, and $\delta \leq y-x \leq y-x+u \leq y-x+\delta$ by Eq. 5 and the property $(\mathbf{H})$ we have

$$
G_{(0, \infty)}(x, y)=\int_{0}^{x} V^{\prime}(u) V^{\prime}(y-x+u) d u \leq H V^{\prime}(y-x) V(x) .
$$

Next, since $\delta<y-2 \delta+u \leq y-x \leq y-2 \delta+u+2 \delta$ for $0 \leq u \leq \delta$, using again the property $(\mathbf{H})$ we arrive at

$$
V^{\prime}(y-x) \leq H V^{\prime}(y-2 \delta+u)
$$


Multiplying both sides by $V^{\prime}(u)$ and integrating over $[0, \delta]$ we obtain

$V^{\prime}(y-x) V(\delta) \leq H \int_{0}^{\delta} V^{\prime}(y-2 \delta+u) V^{\prime}(u) d u \leq H \int_{0}^{2 \delta} V^{\prime}(y-2 \delta+u) V^{\prime}(u) d u=H G_{(0, \infty)}(2 \delta, y)$,

which completes the proof.

According to [2, Theorem VI.20] we have for any non-negative function $f$ on $[0, \infty)$,

$$
\int_{0}^{\infty} f(y) G_{(0, \infty)}(x, y) d y=\int_{0}^{\infty} V^{\prime}(y) d y \int_{0}^{x} f(x+y-u) V^{\prime}(u) d u .
$$

Applying this to the indicator of the interval $[0, z], z>0$, we have $M(x, z)=$ $\int_{0}^{z} G_{(0, \infty)}(x, y) d y=\int_{0}^{z} V^{\prime}(y) d y \int_{(x+y-z) \vee 0}^{x} V^{\prime}(u) d u$. It is then clear that

$$
V(z-x) V(x) \leq M(x, z) \leq V(x) V(z), \quad 0<x \leq z<\infty .
$$

Lemma 4.7 Suppose that $(\boldsymbol{H})$ holds. Let $F(z)$ be non-negative subadditive on $\mathbb{R}$ and $\mathbb{E}^{x} F\left(X_{\tau_{(0, \infty)}}\right) \leq F(x), x>0$. Then, for $0<x<1$,

$$
\mathbb{E}^{x}\left[X_{\tau_{(0, \infty)}} \leq-2 ; F\left(X_{\tau_{(0, \infty)}}\right)\right] \leq c H^{2} F^{*}(1) \frac{V(x)}{V(1)},
$$

where $F^{*}(x)=\sup _{|z| \leq|x|} F(z)$. The constant $c$ is absolute.

Proof By the Ikeda-Watanabe formula,

$$
\mathbb{E}^{x}\left[X_{\tau_{(0, \infty)}} \leq-2 ; F\left(X_{\left.\tau_{(0, \infty)}\right)}\right)\right]=\int_{-\infty}^{-2} F(z) \int_{0}^{\infty} G_{(0, \infty)}(x, y) v(z, d y) d z .
$$

By Lemma 4.6,

$$
G_{(0, \infty)}(x, y) \leq H^{2} \frac{V(x)}{V(2)} G_{(0, \infty)}(4, y), \quad x \leq 2<6 \leq y
$$

Hence,

$$
\int_{6}^{\infty} G_{(0, \infty)}(x, y) v(z, d y) \leq H^{2} \frac{V(x)}{V(2)} \int_{6}^{\infty} G_{(0, \infty)}(4, y) v(z, d y) .
$$

Note that $\mathbb{E}^{z} F\left(X_{\tau_{(0, \infty)}}\right) \leq$ for $z>0 F(z)$, which implies

$$
I=\int_{-\infty}^{-2} F(z) \int_{6}^{\infty} G_{(0, \infty)}(x, y) v(z, d y) d z \leq H^{2} \frac{V(x)}{V(2)} \mathbb{E}^{4} F\left(X_{\tau_{(0, \infty)}}\right) \leq H^{2} \frac{V(x)}{V(2)} F(4) .
$$

Observe that by subadditivity of $F, F(w+y) \leq F(w)+F(y) \leq F(w)+F^{*}(6)$ if $0<y<6$ and $w<-2-y$. By Eq. 18 we have $\int_{0}^{6} G_{(0, \infty)}(x, y) d y \leq V(6) V(x) \leq 2 V(4) V(x)$, hence

$$
\begin{aligned}
I I & =\int_{-\infty}^{-2} F(z) \int_{0}^{6} G_{(0, \infty)}(x, y) v(z, d y) d z=\int_{0}^{6} G_{(0, \infty)}(x, y) \int_{-\infty}^{-2-y} F(w+y) v(d w) d y \\
& \leq 2 V(x) \frac{V(2) V(4)}{V(2)} \int_{-\infty}^{-2} F(w) v(d w)+2 V(x) F^{*}(6) V(4) v([2, \infty)) .
\end{aligned}
$$


Note that by Eq. 18, $V(2) V(4) \leq \int_{0}^{6} G_{(0, \infty)}(2, y) d y$, and $F(w-y) \leq F(w)+F^{*}(6)$ if $0<y<6$ and $w<-6$ which imply

$$
\begin{aligned}
V(2) V(4) \int_{-\infty}^{-12} F(w) v(d w) & \leq \int_{0}^{6} \int_{-\infty}^{-12} F(w) G_{(0, \infty)}(2, y) v(d w) d y \\
& \leq \int_{0}^{6} \int_{-\infty}^{-6-y} F(w) G_{(0, \infty)}(2, y) v(d w) d y \\
& =\int_{0}^{6} \int_{-\infty}^{-6} F(w-y) G_{(0, \infty)}(2, y) v(y, d w) d y \\
& \leq \int_{0}^{6} \int_{-\infty}^{-6}\left(F(w)+F^{*}(6)\right) G_{(0, \infty)}(2, y) v(y, d w) d y \\
& \leq \mathbb{E}^{2} F\left(X_{\tau_{(0, \infty)}}\right)+F^{*}(6) \mathbb{P}^{2}\left(X_{\tau_{(0, \infty)}} \leq-6\right) \\
& \leq F(2)+F^{*}(6) .
\end{aligned}
$$

Next, by Lemma 2.9,

$$
\int_{-12}^{-2} F(w) v(d w) \leq F^{*}(12) v[2, \infty) \leq C_{2} \frac{F^{*}(12)}{V^{2}(2)} .
$$

Combining all the estimates obtained above and using subaddativity of $F^{*}$ and $V$ we conclude that there is an absolute constant $c$ such that

$$
\mathbb{E}^{x}\left[X_{\tau_{(0, \infty)}} \leq-2 ; F\left(X_{\left.\tau_{(0, \infty)}\right)}\right)=I+I I \leq c H^{2} F^{*}(1) \frac{V(x)}{V(1)}, \quad 0<x<1,\right.
$$

which ends the proof.

Lemma 4.8 Let $\psi \in W L S C(\alpha, \gamma), \alpha>1$ and let $F$ be a non-negative harmonic function on $(0,2 R), R>0$. Suppose that $r>0$ is such that $V(R) \geq 2 V(r) / C_{4}$, where $C_{4}$ is the constant from Lemma 2.11. Then for $0<x<r$,

$$
\frac{F(x)}{F(r)} \geq \frac{C_{4}}{2}\left(C_{H}\right)^{R / r+1} \frac{V(x)}{V(r)},
$$

where $C_{H}$ is the constant from the Harnack inequality (17), which depends only on the scalings.

Proof Observe that $V(R) \geq 2 V(r) / C_{4}$ implies that $V(R)>V(r)$, hence $r<R$. Since $F$ is harmonic then using the Harnack inequality (Theorem 4.5) we have for every $r \leq x, y \leq$ $R$ such that $|x-y|<r, F(x) \geq C_{H} F(y)$. By the chaining argument we have for any $r \leq x \leq R$

$$
F(x) \geq\left(C_{H}\right)^{R / r+1} F(r) .
$$

By Lemma 2.11,

$$
\begin{aligned}
\mathbb{P}^{x}\left(X_{\tau_{(0, r)}} \in[r, R)\right) & =\mathbb{P}^{x}\left(X_{\tau_{(0, r)}} \geq r\right)-\mathbb{P}^{x}\left(X_{\tau_{(0, r)}} \geq R\right) \geq \mathbb{P}^{x}\left(X_{\tau_{(0, r)}} \geq r\right)-\mathbb{P}^{x}\left(X_{\tau_{(0, R)}} \geq R\right) \\
& \geq C_{4} \frac{V(x)}{V(r)}-\frac{V(x)}{V(R)} \geq \frac{C_{4}}{2} \frac{V(x)}{V(r)}
\end{aligned}
$$


Note that by Eq. 19, quasi left-continuity of $X$ and harmonicity of $F$,

$$
\begin{aligned}
F(x) & =\lim _{\varepsilon \rightarrow 0^{+}} \mathbb{E}^{x} F\left(X_{\tau_{(\varepsilon, r)}}\right) \geq \lim _{\varepsilon \rightarrow 0^{+}} \mathbb{E}^{x}\left[F\left(X_{\left.\tau_{(\varepsilon, r)}\right)}, X_{\tau_{(\varepsilon, r)}} \in[r, R]\right]\right. \\
& \geq C_{H}^{R / r+1} F(r) \lim _{\varepsilon \rightarrow 0^{+}} \mathbb{P}^{x}\left(X_{\tau_{(\varepsilon, r)}} \in[r, R]\right)=C_{H}^{R / r+1} F(r) \mathbb{P}^{x}\left(X_{\tau_{(0, r)}} \in[r, R]\right) \\
& \geq \frac{C_{H}^{R / r+1} C_{4}}{2} F(r) \frac{V(x)}{V(r)} .
\end{aligned}
$$

\section{Hitting Times of Intervals}

Throughout this section $B_{R}=[-R, R], R>0$. The goal is to find sharp estimates for the tail function of $T_{B_{R}}$ and we start with the case $R=1$. Once this is done we use the scaling argument to treat any $R>0$. The proposition below provides an effective tool for the upper bound.

Proposition 5.1 Suppose that the condition $(\boldsymbol{H})$ holds. If $1>1 / \psi^{*}(1)$, then

$$
\mathbb{P}^{x}\left(T_{B_{1}}>1\right) \leq c H^{2} \frac{V(|x|-1)}{V(|x|)}\left[\sup _{|z| \leq 1} \mathbb{P}^{z}\left(T_{0}>1 / 2\right)+\mathbb{P}^{x-1}\left(T_{0}>1 / 2\right)\right], \quad|x|>1 .
$$

The constant $c$ is absolute.

Proof If $|x| \geq 2$ we have, by subadditivity of $V, \frac{V(|x|-1)}{V(|x|)} \geq \frac{1}{3}$, hence the conclusion is obvious since $H \geq 1$ and $\mathbb{P}^{x}\left(T_{B_{1}}>1\right) \leq \mathbb{P}^{x-1}\left(T_{0}>1 / 2\right)$.

Let $1<x<2$. The condition $1>1 / \psi^{*}(1)$, by Lemma 2.8 , implies $1 / V(1) \leq C_{1}$. Then, by Lemma 2.10 and subadditivity of $V$,

$$
\mathbb{P}^{x}\left(\tau_{(1, \infty)}>1 / 2\right) \leq 2 \sqrt{2} \frac{C_{1}}{C_{3}} \frac{V(x-1)}{V(1)} \mathbb{P}^{2}\left(\tau_{(1, \infty)}>1\right) \leq 4 \sqrt{2} \frac{C_{1}}{C_{3}} \frac{V(x-1)}{V(x)} \mathbb{P}^{1}\left(T_{0}>1 / 2\right) .
$$

Since

$$
\mathbb{P}^{x}\left(T_{B_{1}}>1\right) \leq \mathbb{P}^{x}\left(\tau_{(1, \infty)}>1 / 2\right)+\mathbb{E}^{x} \mathbb{P}^{X_{\tau_{(1, \infty)}}}\left(T_{B_{1}}>1 / 2\right)
$$

it is enough to estimate the harmonic function

$$
\begin{aligned}
\mathbb{E}^{x} \mathbb{P}^{X_{\tau_{(1, \infty)}}\left(T_{B_{1}}>1 / 2\right)} \leq \mathbb{E}^{x}\left[X_{\tau_{(1, \infty)}} \leq-1 ; \mathbb{P}^{\left.X_{\tau_{(1, \infty)}}\left(T_{1}>1 / 2\right)\right]}\right. \\
=\mathbb{E}^{x-1}\left[X_{\tau_{(0, \infty)}} \leq-2 ; \mathbb{P}^{X_{\tau_{(0, \infty)}}}\left(T_{0}>1 / 2\right)\right] .
\end{aligned}
$$

Let $F(z)=\mathbb{P}^{z}\left(T_{0}>1 / 2\right)$. Observe that this function is subadditive and satisfies the assumptions of Lemma 4.7. Therefore the conclusion follows from Lemma 4.7.

Corollary 5.2 Let $\psi \in W L S C(\alpha, \gamma), \alpha>1$. If $|x|>1$ and $1>1 / \psi^{*}(1)$ then

$$
\mathbb{P}^{x}\left(T_{B_{1}}>1\right) \leq c \frac{V(|x|-1) K(|x|)}{V(|x|) K\left(1 / \psi^{-1}(1)\right)} \wedge 1 \approx \frac{V(|x|-1) K(|x|)}{V(|x|) \psi^{-1}(1)} \wedge 1 .
$$

The constant $c$ depends only on the scalings.

Proof By Remark 3 we find a constant $H$ dependent only on the scalings such that the property $(\mathbf{H})$ holds. Therefore, by applying Propositions 5.1 and 3.1 together with Lemma 2.14 we end the proof. 
Remark 4 If $\mathbb{P}^{x}\left(T_{0}>t\right) \leq c\left(\frac{K(x)}{K\left(1 / \psi^{-1}(1 / t)\right)} \wedge 1\right)$ the WLSC assumption is merely to assure the property $(\mathbf{H})$. However there are many examples for which $V^{\prime}$ is non-increasing and then this property holds automatically with the constant $H=1$. For example if $X$ is a special subordinate Brownian motion satisfying (1), then the estimate from the preceding corollary holds with an absolute constant. In particular $\psi(x)=|x|+|x|^{2}$ defines a special subordinate Brownian motion and it does not have the lower scaling property with index $\alpha>1$.

Next, we deal with the lower bound.

Proposition 5.3 Let $\psi \in W L S C(\alpha, \gamma), \alpha>1$, and let $1>1 / \psi^{*}(1)$. There is $x^{*} \geq 2$, which depends only on the scaling characteristics, such that for $|x| \geq x^{*}$ we have

$$
\mathbb{P}^{x}\left(T_{B_{1}}>2\right) \geq C_{5}\left(\frac{K(|x|)}{K\left(1 / \psi^{-1}(1)\right)} \wedge 1\right) \approx\left(\frac{K(|x|)}{\psi^{-1}(1)} \wedge 1\right) .
$$

The constant $C_{5}$ depends only on the scalings.

Proof By symmetry we may assume that $x>0$. Let $f(t)=\mathbb{P}^{x}\left(T_{B_{1}}>t\right), f_{0}(t)=$ $\mathbb{P}^{x}\left(T_{0}>t\right)$. We begin with a simple observation relating the Laplace transforms of $f(t)$ and $f_{0}(t)$. By Eq. 16,

$$
\begin{aligned}
\lambda u^{\lambda}(0) \mathcal{L} f(\lambda) & =K^{\lambda}(x)-\mathbb{E}^{x} e^{-\lambda T_{B_{1}}} K^{\lambda}\left(X_{T_{B_{1}}}\right) \\
& \geq K^{\lambda}(x)-\mathbb{E}^{x} K\left(X_{T_{B_{1}}}\right) \\
& =\lambda u^{\lambda}(0) \mathcal{L} f_{0}(\lambda)-\mathbb{E}^{x} K\left(X_{T_{B_{1}}}\right) .
\end{aligned}
$$

Let $\gamma(\theta, z)=\int_{0}^{z} u^{\theta-1} e^{-u} d u, z>0, \theta>0$ be the lower incomplete Gamma function of index $\theta$. We pick $0<b \leq 1$ to be specified later. By [7, Lemma 5], $\gamma(1,1) f_{0}(s) \leq$ $\mathcal{L} f_{0}\left(s^{-1}\right) s^{-1}, s>0$. Moreover, Eq. 15 implies $\mathcal{L} f_{0}\left(s^{-1}\right) \leq c_{1}(\lambda s)^{1 / \alpha} \mathcal{L} f_{0}(\lambda), s \leq \lambda^{-1}$, where $c_{1}$ depends on the scalings. Hence

$$
\begin{aligned}
\lambda \mathcal{L} f(\lambda) & =\lambda \int_{0}^{b \lambda^{-1}} e^{-\lambda s} f(s) d s+\lambda \int_{b \lambda^{-1}}^{\infty} e^{-\lambda s} f(s) d s \\
& \leq \frac{\lambda}{\gamma(1,1)} \int_{0}^{b \lambda^{-1}} e^{-\lambda s} \mathcal{L} f_{0}\left(s^{-1}\right) s^{-1} d s+f\left(b \lambda^{-1}\right) \int_{b \lambda^{-1}}^{\infty} e^{-\lambda s} \lambda d s \\
& \leq \frac{\lambda}{\gamma(1,1)} \int_{0}^{b \lambda^{-1}} c_{1}(\lambda s)^{1 / \alpha} \mathcal{L} f_{0}(\lambda) e^{-\lambda s} s^{-1} d s+f\left(b \lambda^{-1}\right) e^{-b} \\
& =c_{1} \frac{\gamma(1 / \alpha, b)}{\gamma(1,1)} \lambda \mathcal{L} f_{0}(\lambda)+f\left(b \lambda^{-1}\right) e^{-b}
\end{aligned}
$$

Next we choose the largest $b \leq 1$ such that $2 c_{1} \gamma(1 / \alpha, b) \leq \gamma(1,1)=1-e^{-1}$. Since $\lambda \mathcal{L} f(\lambda) \geq \lambda \mathcal{L} f_{0}(\lambda)-\frac{\mathbb{E}^{x} K\left(X_{T_{B_{1}}}\right)}{u^{\lambda}(0)}$, then

$$
f\left(b \lambda^{-1}\right) \geq \lambda \mathcal{L} f_{0}(\lambda) / 2-\frac{\mathbb{E}^{x} K\left(X_{T_{B_{1}}}\right)}{u^{\lambda}(0)}=\frac{K^{\lambda}(x)}{2 u^{\lambda}(0)}-\frac{\mathbb{E}^{x} K\left(X_{T_{B_{1}}}\right)}{u^{\lambda}(0)} .
$$


If $x \psi^{-1}(\lambda) \leq 1$ then by Lemma $2.15, K^{\lambda}(x) \geq c_{2} K(x)$, hence in this case

$$
f\left(b \lambda^{-1}\right) \geq \frac{c_{2} K(x)-\mathbb{E}^{x} K\left(X_{T_{B_{1}}}\right)}{u^{\lambda}(0)} .
$$

Letting $\lambda=1 / t$ and applying again Lemma 2.15 to estimate $u^{\lambda}(0)$ we have for $t \geq \frac{1}{\psi^{*}(1 / x)}$,

$$
f(b t) \geq c_{3}\left[\frac{c_{2} K(x)-\sup _{|z| \leq 1} K(z)}{K\left(1 / \psi^{-1}(1 / t)\right)}\right] .
$$

By Lemma 2.14 the lower scaling property with index $\alpha-1$ holds for $K$, therefore we can find $x^{*} \geq 2$, dependent on the scalings, such that $c_{2} K(x)-\sup _{|z| \leq 1} K(z) \geq \frac{c_{2}}{2} K(x), \quad x \geq$ $x^{*}$. Hence, for $x \geq x^{*}$ and $t \geq \frac{1}{\psi^{*}(1 / x)}$ we have

$$
f(b t) \geq c_{4} \frac{K(x)}{K\left(1 / \psi^{-1}(1 / t)\right)} .
$$

For $x \geq x^{*} \geq 2$ and $t \leq \frac{1}{\psi^{*}(1 / x)}$ we apply subaddativity of $V$ and Lemma 2.8 to get $\frac{V(x-1)}{\sqrt{t}} \geq \frac{1}{2} \frac{V(x)}{\sqrt{t}} \geq \frac{1}{2 C_{1}}$. Next, applying Lemma 2.10 to arrive at

$$
f(b t) \geq \mathbb{P}^{x}\left(\tau_{(1, \infty)} \geq b t\right) \geq C_{3}\left(\frac{V(x-1)}{\sqrt{b t}} \wedge 1\right) \geq C_{3}\left(\frac{1}{2 C_{1} \sqrt{b}} \wedge 1\right) .
$$

Therefore we have proved that for $x \geq x^{*}$ and any $t>0$ we have

$$
f(b t) \geq c_{5} \min \left\{\frac{K(x)}{K\left(1 / \psi^{-1}(1 / t)\right)}, 1\right\},
$$

where $c_{5}$ depends on the scalings. In particular taking $t=2 / b$ we obtain

$$
f(2) \geq c_{5} \min \left\{\frac{K(x)}{K\left(1 / \psi^{-1}(b / 2)\right)}, 1\right\} \geq c_{6} \min \left\{\frac{K(x)}{K\left(1 / \psi^{-1}(1)\right)}, 1\right\}, \quad|x| \geq x^{*},
$$

where the last inequality follows from scaling property for $K$ and $\psi^{-1}$ (see Lemma 2.14 and Eq. 10). The constant $c_{6}$ depends on the scalings.

Lemma 5.4 Let $\psi \in \operatorname{WLSC}(\alpha, \gamma), \alpha>1$. If $1<|x|<x^{*}$ and $1>1 / \psi^{*}(1)$, where $x^{*}$ is chosen in the preceding lemma, then

$$
\mathbb{P}^{x}\left(T_{B_{1}}>1\right) \geq c \frac{V(|x|-1) K(|x|)}{V(|x|) K\left(1 / \psi^{-1}(1)\right)} \wedge 1 .
$$

The constant $c$ depends only on the scalings.

Proof We may and do assume that $1 \leq x<x^{*}$. By the strong Markov property we have for any $z \geq 1$,

$$
\mathbb{P}^{z}\left(T_{B_{1}}>2\right) \leq \mathbb{P}^{z}\left(\tau_{(1, \infty)}>1\right)+\mathbb{E}^{z} \mathbb{P}^{X_{\tau(1, \infty)}}\left(T_{B_{1}}>1\right)
$$

and

$$
\mathbb{P}^{z}\left(T_{B_{1}}>1\right) \geq \frac{1}{2} \mathbb{P}^{z}\left(\tau_{(1, \infty)}>1\right)+\frac{1}{2} \mathbb{E}^{z} \mathbb{P}^{X_{\tau_{(1, \infty)}}}\left(T_{B_{1}}>1\right) .
$$

Using Lemma 4.8 we estimate the harmonic function $F(z)=\mathbb{E}^{z} \mathbb{P}^{X_{\tau_{(1, \infty)}}}\left(T_{B_{1}}>1\right)$,

$$
F(x) \geq c_{1} \frac{V(x-1)}{V(2)} F\left(x^{*}\right),
$$


with the constant $c_{1}$ dependent only on the scalings. From Lemma 2.10 and subaddativity of $V$ we infer that $\mathbb{P}^{x}\left(\tau_{(1, \infty)}>1\right) \geq c_{2} \frac{V(x-1)}{V(2)} \mathbb{P}^{x^{*}}\left(\tau_{(1, \infty)}>1\right)$, with $c_{2}$ dependent only on the scalings. Hence,

$$
\begin{aligned}
\mathbb{P}^{x}\left(T_{B_{1}}>1\right) & \geq 1 / 2\left(\mathbb{P}^{x}\left(\tau_{(1, \infty)}>1\right)+\mathbb{E}^{x} \mathbb{P}^{X_{\tau_{(1, \infty)}}}\left(T_{B_{1}}>1\right)\right) \\
& \geq \frac{c_{1} \wedge c_{2}}{2} \frac{V(x-1)}{V(2)}\left(\mathbb{P}^{x^{*}}\left(\tau_{(1, \infty)}>1\right)+\mathbb{E}^{x^{*}} \mathbb{P}^{X_{\tau}(1, \infty)}\left(T_{B_{1}}>1\right)\right) \\
& \geq \frac{c_{1} \wedge c_{2}}{2} \frac{V(x-1)}{V(2)} \mathbb{P}^{x^{*}}\left(T_{B_{1}}>2\right) .
\end{aligned}
$$

Applying Proposition 5.3 we get $\mathbb{P}^{x^{*}}\left(T_{B_{1}}>2\right) \geq C_{5}\left(\frac{K(2)}{K\left(1 / \psi^{-1}(1)\right)} \wedge 1\right)$, which completes the proof.

Now we are ready to state and prove the main result of this section.

Theorem 5.5 Let $\psi \in W L S C(\alpha, \gamma), \alpha>1$. If $B_{R}=[-R, R]$ and $|x|>R$,

$$
\mathbb{P}^{x}\left(T_{B_{R}}>t\right) \approx \frac{V(|x|-R)}{\sqrt{t} \wedge V(R)} \wedge 1, \quad t \leq 1 / \psi^{*}(1 / R)
$$

and

$\mathbb{P}^{x}\left(T_{B_{R}}>t\right) \approx \frac{V(|x|-R) K(|x|)}{V(|x|) K\left(1 / \psi^{-1}(1 / t)\right)} \wedge 1 \approx \frac{V(|x|-R) K(|x|)}{V(|x|) t \psi^{-1}(1 / t)} \wedge 1, \quad t>1 / \psi^{*}(1 / R)$.

The comparability constants depend only on the scaling characteristics.

Proof If $t \leq 1 / \psi^{*}(1 / R)$ the estimates hold by [5, Remark 6] and Lemma 2.10.

Let $t>0, R>0$ be fixed. We consider a space and time rescaled process $Y_{S}=$ $X_{t s} / R, s \geq 0$. Let $K_{R}^{t}$, etc. be objects corresponding to the process $Y$. Then

$$
\begin{aligned}
\psi_{R}^{t}(x) & =t \psi(x / R), \\
K_{R}^{t}(x) & =\frac{R}{t} K(x R), \\
V_{R}^{t}(x) & =\frac{V(x R)}{\sqrt{t}}, \\
\psi_{R}^{-1}(x) & =R \psi^{-1}(x / t) .
\end{aligned}
$$

Let $T_{B_{1}}^{Y}$ be the hitting time of $B_{1}$ by the process $Y$. Observe that $\psi_{R}^{t}(x)$ has exactly the same scaling property (with the same scaling characteristics) as $\psi(x)$. Let $t>1 / \psi^{*}(1 / R)$ or equivalently $1>1 /\left(\psi_{R}^{t}\right)^{*}(1)$. We now apply Corollary 5.2, Proposition 5.3 and Lemma 5.4 to get

$$
\begin{aligned}
\mathbb{P}^{x}\left(T_{B_{R}}>t\right) & =\mathbb{P}^{x / R}\left(T_{B_{1}}^{Y}>1\right) \\
& \approx \frac{V_{R}^{t}(|x / R|-1) K_{R}^{t}(|x / R|)}{V_{R}^{t}(|x / R|) K_{R}^{t}\left(1 /\left(\psi_{R}^{t}\right)^{-1}(1)\right)} \wedge 1 \\
& =\frac{V(|x|-R) K(|x|)}{V(|x|) K\left(1 / \psi^{-1}(1 / t)\right)} \wedge 1 \\
& \approx \frac{V(|x|-R) K(|x|)}{V(|x|) t \psi^{-1}(1 / t)} \wedge 1,
\end{aligned}
$$

where the comparability constants depend only on the scalings of $\psi$. 
Corollary 5.6 Assume that the second moment of $X$ is finite and $\psi \in W L S C(\alpha, \gamma), \alpha>1$. Let $R>1$ and $B_{R}=[-R, R]$. Then

$$
\mathbb{P}^{x}\left(T_{B_{R}}>t\right) \approx \frac{V(|x|-R)}{\sqrt{t}} \wedge 1, \quad t>0 .
$$

The comparability constant depends on $\psi$.

Proof If the second moment is finite, then $\psi(x) \approx x^{2},|x|<1$, hence $\psi^{-1}(x) \approx \sqrt{x}, 0 \leq$ $x<1$. Moreover $K(w) \approx V(w) \approx w, w>1$. Hence we obtain the conclusion applying Theorem 5.5.

Remark 5 If $X$ is a special subordinate Brownian motion satisfying (1), then the upper bound from Theorem 5.5 is true without the assumption $\psi \in \operatorname{WLSC}(\alpha, \gamma), \alpha>1$. This follows from the fact that property $(\mathbf{H})$ holds for such processes (see Remark 3). In particular $\psi(x)=|x|+|x|^{2}$ defines a special subordinate Brownian and it does not have a lower scaling property with $\alpha>1$ but we have

$$
\mathbb{P}^{x}\left(T_{B_{R}}>t\right) \leq c \frac{V(|x|-R)}{\sqrt{t} \wedge V(R)} \wedge 1, \quad t \leq 1 / \psi^{*}(1 / R),
$$

and

$$
\mathbb{P}^{x}\left(T_{B_{R}}>t\right) \leq c \frac{V(|x|-R) K(|x|)}{V(|x|) K\left(1 / \psi^{-1}(1 / t)\right)} \wedge 1, \quad t>1 / \psi^{*}(1 / R) .
$$

Here the constant $c$ is independent of $R, V(x) \approx \sqrt{x} \wedge x, x \geq 0$ and $K(x) \approx \log (1+$ $|x|), x \in \mathbb{R}$.

By inspecting the proof of Proposition 5.3 it is clear that we can prove a lower bound

$$
\mathbb{P}^{x}\left(T_{B_{R}}>t\right) \geq c_{R} \frac{V(|x|-R) K(|x|)}{V(|x|) K\left(1 / \psi^{-1}(1 / t)\right)} \wedge 1, \quad t>1 / \psi^{*}(1 / R),
$$

but the constant $c_{R}$ will be dependent on $R$ (to choose $x^{*}$ as in Proposition 5.3 one can use unboundedness of $K$ instead of the scaling property).

Example 4 Let $v(r)=\frac{1}{|r|^{3} \log ^{2}(2+1 /|r|)}, r \in \mathbb{R}$. Since $v$ is decreasing on $(0, \infty)$, by Lemma 2.12, $\psi(x) \approx \int_{0}^{\infty}\left(1 \wedge(x r)^{2}\right) v(r) d r=x^{2} \int_{0}^{1 / x} r^{2} v(r) d r+\int_{1 / x}^{\infty} v(r) d r$. Elementary calculations show that

$$
\begin{gathered}
\psi(x) \approx x^{2} \frac{\log (2+1 / x)}{\log (2+x)}, \quad x>0, \\
\psi^{-1}(x) \approx \sqrt{x} \frac{\sqrt{\log (2+x)}}{\sqrt{\log (2+1 / x)}}, \quad x>0 .
\end{gathered}
$$

It is clear that $\psi$ has the weak lower scaling property with any $1<\alpha<2$. Hence

$$
K(x) \approx \frac{1}{x \psi(1 / x)} \approx x \frac{\log (2+1 / x)}{\log (2+x)}, \quad x>0
$$

and

Hence

$$
V(x) \approx \frac{1}{\sqrt{\psi(1 / x)}} \approx x \frac{\sqrt{\log (2+1 / x)}}{\sqrt{\log (2+x)}}, \quad x>0 .
$$

$$
\frac{K(x)}{V(x)} \approx \frac{\sqrt{\log (2+1 / x)}}{\sqrt{\log (2+x)}}, \quad x>0 .
$$


Applying Theorem 5.5 we obtain for $|x|>R$,

$$
\mathbb{P}^{x}\left(T_{B_{R}}>t\right) \approx(|x|-R) \frac{\sqrt{\log (2+1 /(|x|-R))}}{\sqrt{t \log (2+(|x|-R))}} \wedge 1, \quad t \leq 1 / \psi^{*}(1 / R)
$$

and for $t>1 / \psi^{*}(1 / R)$,

$$
\mathbb{P}^{x}\left(T_{B_{1}}>t\right) \approx(|x|-R) \frac{\sqrt{\log (2+1 /(|x|-R))}}{\sqrt{\log (2+(|x|-R))}} \frac{\sqrt{\log (2+1 /|x|)}}{\sqrt{\log (2+|x|)}} \frac{\sqrt{\log (2+t)}}{\sqrt{t \log (2+1 / t)}} \wedge 1,
$$

since $t \psi^{-1}(1 / t) \approx \sqrt{t \frac{\log (2+1 / t)}{\log (2+t)}}$.

\section{Heat Kernel Estimates}

This section is devoted to finding sharp estimates of the heat kernel of the process $X$ killed after hitting an interval. We apply the previous results on hitting times and the estimates of the heat kernel of the free process obtained in [7] under the assumption of unimodality of $X$ and both lower and upper scaling property of $\psi$. At the end of the section we suggest a certain extension of the main result, which allows to treat symmetric processes which are not unimodal.

We denote $D_{R}=(-R,-1) \cup(1, R), R>1$.

Proposition 6.1 Let $\psi \in W L S C(\alpha, \gamma), \alpha>1$. For $|x| \in(1, R)$ and $R>1$ we have

$$
\mathbb{E}^{x} \tau_{D_{R}} \leq C_{6} R \frac{V(|x|-1) K(|x|)}{V(|x|)},
$$

where the constant $C_{6}$ depends on the scalings.

Proof Let $x>1$. By Proposition 2.4,

$$
\begin{aligned}
\mathbb{E}^{x} \tau_{D_{R}} & =\int_{(-R,-1) \cup(1, R)} G_{(-R,-1) \cup(1, R)}(x, y) d y \leq 2 \int_{0}^{R} G_{\{0\}^{c}}(x-1, y) d y \\
& \leq 4 R K(x-1),
\end{aligned}
$$

which gives the desired bound if $x>2$, since $\frac{V(x-1)}{V(x)} \geq 1 / 2$.

Assume that $1<x \leq 2$. Let $s(u)=\mathbb{E}^{u} \tau_{D_{R}}, u \in \mathbb{R}$. Then by the strong Markov property we have

$$
s(x)=\mathbb{E}^{x} \tau_{(1, R)}+\mathbb{E}^{x} s\left(X_{\tau_{(1, R)}}\right) .
$$

Next, applying the above estimate and subaddativity of $K$ we obtain

$$
\begin{aligned}
\mathbb{E}^{x} s\left(X_{\tau_{(1, R)}}\right) & \leq 4 R \mathbb{E}^{x}\left[K\left(\left|X_{\tau_{(1, R)}}\right|-1\right) ; X_{\tau_{(1, R)}} \leq-1\right] \\
& \leq 4 R \mathbb{E}^{x}\left[K\left(X_{\tau_{(1, R)}}\right)+K(1) ; X_{\tau_{(1, R)}} \leq-1\right] \\
& \leq 4 R \mathbb{E}^{x}\left[K\left(X_{\tau_{(1, \infty)}}\right)+K(1) ; X_{\tau_{(1, \infty)}} \leq-1\right] \\
& =4 R \mathbb{E}^{x-1}\left[K\left(X_{\tau_{(0, \infty)}}\right)+K(1) ; X_{\tau_{(0, \infty)}} \leq-2\right] \\
& \leq c_{1} R \frac{V(x-1) K^{*}(1)}{V(1)}
\end{aligned}
$$


where in the last step we applied Lemma 4.1 and Lemma 4.7. Note that the constant $c_{1}$ depends only on the scalings. Finally, applying [22, Proposition 3.5], subadditivity of $V$ and the estimate $V^{2}(2) \leq c_{2} K(2)$ following from Lemma 2.14 , we obtain

$$
\mathbb{E}^{x} \tau_{(1, R)} \leq 2 V(x-1) V(R) \leq 2 R V(x-1) V(2) \leq c_{2} R \frac{V(x-1) K(2)}{V(2)} .
$$

The proof is completed by observing that, by Lemma $2.14, K^{*}(1) \approx K(|x|), 1 \leq|x| \leq 2$ and by subaddativity $V(1) \approx V(|x|), 1 \leq|x| \leq 2$.

Proposition 6.2 Let $R>2$ and $1<|x|<R$. If $\psi \in W L S C(\alpha, \gamma), \alpha>1$, then

$$
\mathbb{P}^{x}\left(\left|X_{\tau_{D_{R}}}\right| \geq R\right) \leq C_{7} \frac{V(|x|-1)}{V(|x|)} \frac{K(|x|)}{K(R)},
$$

where the constant $C_{7}$ depends only on the scalings.

Proof By subaddativity of $V$ and $K$ it is enough to consider $1<x \leq(R \vee 3) / 2$. By Eq. 9 , and then Proposition 6.1,

$$
\mathbb{P}^{x}\left(\left|X_{\tau_{D_{R}}}\right| \geq R\right) \leq C_{2} \frac{\mathbb{E}^{x} \tau_{D_{R}}}{V^{2}(R)} \leq C_{2} C_{6} R \frac{V(x-1) K(x)}{V(x) V^{2}(R)} .
$$

The proof is completed by observing that $K(R) \approx V^{2}(R) / R$ with the comparability constant dependent only on the scalings, which folows from Lemma 2.14 .

The following lemma is consequence of [33, (3.2)], [21, Corollary 1] and the weak lower scaling property.

Lemma 6.3 Let $\psi \in \operatorname{WLSC}(\alpha, \gamma), \alpha>0$. There is a constant $C_{8} \geq 1$ dependent on the scalings such that for $R=\chi / \psi^{-1}(1), \chi>1$, we have

$$
\mathbb{P}^{0}\left(\tau_{(-R, R)} \leq 1\right) \leq \frac{C_{8}}{\chi^{\alpha}} .
$$

In the next proposition we prove estimates for some exit times which play a crucial role in obtaining the main result of this section. Recall that $T_{B_{1}}$ is the first hitting time of $B_{1}$.

Proposition 6.4 Let $\psi \in W L S C(\alpha, \gamma), \alpha>1$. Assume that $1>1 / \psi^{*}(1)$. There is $\chi \geq 2$ dependent only on the scalings such that for $R=\frac{\chi}{\psi^{-1}(1)}>2$ and $D_{R}=(-R,-1) \cup(1, R)$ we have

Moreover

$$
\mathbb{P}^{x}\left(\tau_{D_{R}}>1\right) \geq C_{9} \mathbb{P}^{x}\left(T_{B_{1}}>1\right), \quad 1<|x| \leq \frac{R}{2}
$$

$$
\mathbb{P}^{0}\left(\tau_{(-R / 4, R / 4)}>1\right) \geq 1 / 2
$$

The constant $C_{9}$ depends only on the scalings.

Proof First observe that $1>1 / \psi^{*}(1)$ is equivalent to $\frac{1}{\psi^{-1}(1)}>1$. Let $x^{*}$ be the value picked in Proposition 5.3. We first consider $|x| \geq x^{*} \geq 2$. By Proposition 5.3,

$$
\mathbb{P}^{x}\left(T_{B_{1}}>2\right) \geq C_{5}\left(\frac{K(|x|)}{K\left(1 / \psi^{-1}(1)\right)} \wedge 1\right) .
$$


We find $1 \leq \chi_{1} \leq \chi / 4$ satisfying the following conditions

$$
\begin{aligned}
\frac{K\left(1 / \psi^{-1}(1)\right)}{K\left(\chi / \psi^{-1}(1)\right)} & \leq C_{5} /\left(2 C_{7}\right) \\
\frac{V\left(x^{*}-1\right)}{V\left(\chi / \psi^{-1}(1)\right)} & \leq \frac{1}{8}\left(C_{3}\left(V\left(x^{*}-1\right) \wedge 1\right)\right. \\
\frac{V\left(\chi_{1} / \psi^{-1}(1)\right)}{V\left(\chi / \psi^{-1}(1)\right)} & \leq C_{3} /\left(8 C_{1}\right) \\
\frac{C_{8}}{\chi_{1}^{\alpha}} & \leq \frac{1}{2}
\end{aligned}
$$

Such choice of $\chi_{1}, \chi$, which are dependent on the scalings, is possible due to weak lower scaling property for $V, K$ implied by Lemma 2.8 and Lemma 2.14, respectively.

We set $R=\frac{\chi}{\psi^{-1}(1)}$. The choice of $\chi$ together with Proposition 6.2 imply

$$
\mathbb{P}^{x}\left(\left|X_{\tau_{D_{R}}}\right| \geq R\right) \leq C_{7} \frac{K(|x|)}{K(R)} \leq \frac{C_{5}}{2} \frac{K(|x|-1)}{K\left(1 / \psi^{-1}(1)\right)} .
$$

Then

$$
\mathbb{P}^{x}\left(\tau_{D_{R}}>2\right) \geq \mathbb{P}^{x}\left(T_{B_{1}}>2\right)-\mathbb{P}^{x}\left(\left|X_{\tau_{D_{R}}}\right| \geq R\right) \geq \frac{1}{2} \mathbb{P}^{x}\left(T_{B_{1}}>2\right)
$$

if $x^{*} \leq|x| \leq 1+1 / \psi^{-1}(1)$.

If $1+1 / \psi^{-1}(1)<|x| \leq 1+\chi_{1} / \psi^{-1}(1)$ we use a similar argument based on the exit from a half-space. Indeed, by Lemmas 2.10 and 2.11,

$\mathbb{P}^{x}\left(\tau_{D_{R}}>1\right) \geq \mathbb{P}^{x}\left(\tau_{(1, R)}>1\right) \geq \mathbb{P}^{x}\left(\tau_{(1, \infty)}>1\right)-\mathbb{P}^{x}\left(X_{\tau_{(1, R)}} \geq R\right) \geq C_{3}(V(|x|-1) \wedge 1)-\frac{2 V(|x|-1)}{V(R-1)}$.

Observe that, due to Lemma 2.8, $C_{3}(V(|x|-1) \wedge 1) \geq C_{3}\left(V\left(1 / \psi^{-1}(1)\right) \wedge 1\right) \geq C_{3} / C_{1}$ and by the choice of $\chi_{1}, \frac{2 V(|x|-1)}{V(R-1)} \leq \frac{4 V\left(\chi_{1} / \psi^{-1}(1)\right)}{V(R)} \leq C_{3} /\left(2 C_{1}\right)$. Hence,

$$
\mathbb{P}^{x}\left(\tau_{D_{R}}>1\right) \geq C_{3} /\left(2 C_{1}\right), \quad 1+1 / \psi^{-1}(1)<|x| \leq 1+\chi_{1} / \psi^{-1}(1) .
$$

Next, we assume that $1<|x|<x^{*}$. By Lemmas 2.10, 2.11 and the choice of $R$ we have

$$
\begin{aligned}
\mathbb{P}^{x}\left(\tau_{(1, R)}>1\right) & \geq \mathbb{P}^{x}\left(\tau_{(1, \infty)}>1\right)-\mathbb{P}^{x}\left(X_{\tau_{(1, R)}} \geq R\right) \\
& \geq C_{3}(V(|x|-1) \wedge 1)-\frac{2 V(|x|-1)}{V(R-1)} \\
& =\frac{V(|x|-1)}{V\left(x^{*}-1\right)}\left[\left(C_{3}\left(V\left(x^{*}-1\right) \wedge \frac{V\left(x^{*}-1\right)}{V(x-1)}\right)-\frac{2 V\left(x^{*}-1\right)}{V(R-1)}\right]\right. \\
& \geq \frac{V(|x|-1)}{V\left(x^{*}-1\right)}\left[\left(C_{3}\left(V\left(x^{*}-1\right) \wedge 1\right)-\frac{4 V\left(x^{*}-1\right)}{V(R)}\right]\right. \\
& \geq \frac{V(|x|-1)}{V\left(x^{*}-1\right)} \frac{1}{2} C_{3}\left(V\left(x^{*}-1\right) \wedge 1\right) \\
& \geq \frac{V(|x|-1)}{V\left(x^{*}-1\right)} \frac{1}{4} C_{3} \mathbb{P}^{x^{*}}\left(\tau_{(1, R)}>1\right) .
\end{aligned}
$$


Moreover, we can apply Lemma 4.8 to $F(x)=\mathbb{E}^{x} \mathbb{P}^{X_{\tau_{(1, R)}}}\left(\tau_{D_{R}}>1\right)$ with $r=x^{*}-1$. In consequence we can find $c_{1}$ dependent on the scalings such that

$$
\begin{aligned}
\mathbb{P}^{x}\left(\tau_{D_{R}}>1\right) & \geq \frac{1}{2}\left(\mathbb{P}^{x}\left(\tau_{(1, R)}>1\right)+\mathbb{E}^{x} \mathbb{P}^{X_{\tau_{(1, R)}}}\left(\tau_{D_{R}}>1\right)\right) \\
& \geq c_{1} \frac{V(|x|-1)}{V\left(x^{*}-1\right)}\left(\mathbb{P}^{x^{*}}\left(\tau_{(1, R)}>1\right)+\mathbb{E}^{x^{*}} \mathbb{P}^{X_{\tau_{(1, R)}}}\left(\tau_{D_{R}}>1\right)\right) \\
& \geq c_{1} \frac{V(|x|-1)}{V\left(x^{*}-1\right)} \mathbb{P}^{x^{*}}\left(\tau_{D_{R}}>2\right) \\
& \geq \frac{c_{1}}{2} \frac{V(|x|-1)}{V\left(x^{*}-1\right)} \mathbb{P}^{x^{*}}\left(T_{B_{1}}>2\right),
\end{aligned}
$$

where the last inequality follows from the first part of the proof. Applying Theorem 5.5 we can find $c_{2}$ dependent only on the scalings such that

$$
\mathbb{P}^{x}\left(\tau_{D_{R}}>1\right) \geq \frac{c_{1}}{2} \frac{V(|x|-1)}{V\left(x^{*}-1\right)} \mathbb{P}^{x^{*}}\left(T_{B_{1}}>2\right) \geq c_{2} \mathbb{P}^{x}\left(T_{B_{1}}>1\right),
$$

which completes the proof for $1<|x|<x^{*}$.

Finally we consider $1+\chi_{1} / \psi^{-1}(1) \leq|x| \leq R / 2$. Let $R_{1}=\chi_{1} / \psi^{-1}(1) \leq R / 4$. Then by Lemma 6.3, and the choice of $\chi_{1}$,

$$
\mathbb{P}^{x}\left(\tau_{D_{R}}>1\right) \geq \mathbb{P}^{0}\left(\tau_{\left(-R_{1}, R_{1}\right)}>1\right) \geq 1-\frac{C_{8}}{\chi_{1}^{\alpha}} \geq 1 / 2
$$

and

$$
\mathbb{P}^{0}\left(\tau_{(-R / 4, R / 4)}>1\right) \geq 1 / 2
$$

The proof is completed.

Below we recall optimal estimates for the transition density of an unimodal process $X$ if we assume appropriate scaling conditions.

Lemma 6.5 ([7] ,Corollary 23) Assume that $X$ is unimodal. Let $\psi \in \operatorname{WLSC}(\alpha, \gamma) \cap$ $\operatorname{WUSC}(\beta, \rho), 1<\alpha \leq \beta<2$. Then

$$
p_{t}(x) \approx \psi^{-1}(1 / t) \wedge \frac{t}{|x| V^{2}(|x|)} \approx p_{t}(0) \wedge \frac{t}{|x| V^{2}(|x|)} \approx \psi^{-1}(1 / t) \wedge t v(x), \quad t>0, \quad x, \in \mathbb{R} .
$$

The comparability constants depend on the scalings.

The following lemma is instrumental in estimating the heat kernel $p^{D}$. For the proof see [14, Lemma 2.2] or [6, Lemma 2].

Lemma 6.6 Consider disjoint open sets $U_{1}, U_{3} \subset D$. Let $U_{2}=D \backslash\left(U_{1} \cup U_{3}\right)$. If $x \in U_{1}$, $y \in U_{3}$ and $t>0$, then

$$
\begin{aligned}
& p_{t}^{D}(x, y) \leq \mathbb{P}^{x}\left(X_{\tau_{U_{1}}} \in U_{2}\right) \sup _{s<t, z \in D_{2}} p_{s}(z-y)+\left(t \wedge \mathbb{E}^{x} \tau_{U_{1}}\right) \sup _{u \in U_{1}, z \in U_{3}} v(z-u), \\
& p_{t}^{D}(x, y) \geq t \mathbb{P}^{x}\left(\tau_{U_{1}}>t\right) \mathbb{P}^{y}\left(\tau_{U_{3}}>t\right) \inf _{u \in U_{1}, z \in U_{3}} v(z-u) .
\end{aligned}
$$

Now we are ready to state and prove the main theorem of this section. 
Theorem 6.7 Let $\psi \in(W L S C)(\alpha, \gamma) \cap \operatorname{WUSC}(\beta, \rho), 1<\alpha \leq \beta<2$ and the process is unimodal. Let $D=(-\infty,-r) \cup(r, \infty), r>0$. Then

$$
p_{t}^{D}(x, y) \approx \mathbb{P}^{x}\left(\tau_{D}>t\right) \mathbb{P}^{y}\left(\tau_{D}>t\right) p_{t}(x-y), \quad t>0, x, y \in D .
$$

The comparability constants depend only on the scalings.

Proof We may assume that $|x|<y$. We find the estimates in the case of fixed $t=2$ or $t=3$ and $r=1$, keeping all arising constants dependent only on the scalings. Then applying the scaling argument we will be able to extend the estimates for the whole range of times and any $r>0$. We also assume that $t=1>V^{2}(1)$. The case $1 \leq V^{2}(1)$ can be deduced from a general bound for the killed semigroup obtained in [8, Corollary 2.4, Theorem 3.3 and the beginning of Section 5]. In what follows all comparabilities hold with comparability constants which are either depend only on the scalings or they are absolute. The same remark applies to all constants appearing in the proof. As mentioned above throughout the proof we fix $D=(-\infty,-1) \cup(1, \infty)$.

We start with the upper bound. First, we prove that there is a constant $c_{0}$ such that

$$
p_{1}^{D}(x, y) \leq c_{0} \mathbb{P}^{x}\left(\tau_{D}>2\right) p_{1}(x-y) .
$$

To this end we consider two cases.

Case 1 Assume that $V^{2}(|x-y|) \leq 16$. In this case, by Lemma 6.5, $p_{1}(x-y) \approx p_{1 / 2}(0)$. Hence

$$
\begin{aligned}
p_{1}^{D}(x, y) & =\int p_{1 / 2}^{D}(x, u) p_{1 / 2}^{D}(u, y) d u \\
& \leq p_{1 / 2}(0) \int p_{1 / 2}^{D}(x, u) d u \\
& \leq c_{1} \mathbb{P}^{x}\left(\tau_{D}>1 / 2\right) p_{1}(x-y) .
\end{aligned}
$$

Case 2 Assume that $16<V(|x-y|)$. If $V(|x|-1) \geq 1$ then by Theorem 5.5 (or Lemma 2.10), $\mathbb{P}^{x}\left(\tau_{D}>2\right) \approx 1$ and of course

$$
p_{1}^{D}(x, y) \leq p_{1}(x-y) \leq c_{2} \mathbb{P}^{x}\left(\tau_{D}>2\right) p_{1}(x-y) .
$$

Assume that $V(|x|-1) \leq 1$, and $V(y-1)>2$ (the case $V(|x|-1)<V(y-1)<2$ is included in Case 1). Let $R: V^{2}(R-1)=1$. This implies that $|x| \leq R$. Also, since $1>V^{2}(1)$, we have $R>2$.

We put $U_{1}=(-R,-1) \cup(1, R)=D_{R}$ and $U_{3}=(y-|x-y| / 2, y+|x-y| / 2)$. We claim that $(y-x) / 4 \geq R-x$. Indeed, by subaddativity

$$
V((y-x) / 4) \geq(1 / 4) V(y-x)>4,
$$

while

$$
V(|R-x|) \leq V(R)+V(|x|) \leq V(R-1)+V(|x|-1)+2 V(1) \leq 4 .
$$

Hence

$$
\inf _{u \in U_{1}, z \in U_{3}}|z-u|=y-(y-x) / 2-R \geq(y-x) / 4 .
$$

By Proposition 6.2,

$$
\mathbb{P}^{x}\left(\tau_{U_{1}}<\tau_{D}\right) \leq C_{7} \frac{V(|x|-1) K(|x|)}{V(x) K(R)}, \quad 1<|x|<R .
$$


Since $R \geq 2$ then $1=V(R-1) \leq V(R) \leq 2 V(R-1) \leq 2$, and by Lemma $2.8, R \approx \frac{1}{\psi^{-1}(1)}$. Next, by Lemma $2.14, K(R) \approx \frac{V^{2}(R)}{R} \approx \psi^{-1}(1)$ implies that for all $x: 1<|x|<R$,

$$
\mathbb{P}^{x}\left(\tau_{U_{1}}<\tau_{D}\right) \leq c_{3} \min \left\{\frac{V(|x|-1)}{V(|x|) \psi^{-1}(1)} K(|x|), 1\right\} \leq c_{4} \mathbb{P}^{x}\left(\tau_{D}>1\right),
$$

where the last inequality follows from Theorem 5.5. By Proposition 6.1 and again by Theorem 5.5,

$$
1 \wedge \mathbb{E}^{x} \tau_{U_{1}} \leq C_{6} \min \left\{\frac{V(|x|-1)}{V(|x|) \psi^{-1}(1)} K(|x|), 1\right\} \leq c_{5} \mathbb{P}^{x}\left(\tau_{D}>1\right) .
$$

Let $U_{2}=D \backslash\left(U_{1} \cup U_{3}\right)$. By the estimates of $p_{s}(z-y)$ (see Lemma 6.5),

$$
\sup _{s<1, z \in U_{2}} p_{s}(z-y)=\sup _{s<1} p(s,(x-y) / 2) \leq c_{6} p_{1}(x-y) .
$$

Moreover by Eq. 23,

$$
\sup _{u \in U_{1}, z \in U_{3}} v(z-u) \leq v((x-y) / 4) \approx v(x-y) \leq c_{7} p_{1}(x-y) .
$$

Then, by Lemma 6.6,

$$
\begin{aligned}
p_{1}^{D}(x, y) & \leq\left(1 \wedge \mathbb{E}^{x} \tau_{D_{1}}\right) \sup _{u \in D_{1}, z \in D_{3}} v(z-u)+\sup _{s<1, z \in D_{2}} p_{s}(z-y) \mathbb{P}^{x}\left(\tau_{D_{1}}<\tau_{D}\right) \\
& \leq\left(c_{5} c_{7}+c_{4} c_{6}\right) \mathbb{P}^{x}\left(\tau_{D}>1\right) p_{1}(x-y) .
\end{aligned}
$$

Since, by Theorem $5.5, \mathbb{P}^{x}\left(\tau_{D}>1 / 2\right) \approx \mathbb{P}^{x}\left(\tau_{D}>1\right) \approx \mathbb{P}^{x}\left(\tau_{D}>2\right)$ we arrive at Eq. 20 by combining (21), Eqs. 22 and 24.

Finally, by the semigroup property and by applying the estimate (20), and from symmetry of the heat kernel, we have

$$
p_{2}^{D}(x, y) \leq c_{8} \mathbb{P}^{x}\left(\tau_{D}>2\right) \mathbb{P}^{y}\left(\tau_{D}>2\right) p_{2}(x-y) .
$$

To get a general bound for any $t, r>0$ we consider $Y_{s}=\frac{1}{r} X_{s t}, s \geq 0$. For such a process (fixing $t$ and $r$ ) its characteristic exponent is $\psi_{t}^{r}(u)=t \psi(u / r)$ so it has the same scaling characteristics as $\psi(u)$. Let $p^{Y}, p^{D, Y}$ denote the transition densities for the free and killed process $Y$, respectively. We have $p_{2 t}(x-y)=p_{2}^{Y}((x-y) / r)$ and $p_{2 t}^{r D}(x, y)=$ $p_{2}^{D, Y}(x / r, y / r)$. Moreover $\mathbb{P}^{y / r}\left(\tau_{D}^{Y}>2\right)=\mathbb{P}^{y}\left(\tau_{r D}>2 t\right)$. Hence applying (25) to $Y$ we obtain

$$
\begin{aligned}
p_{r D}(2 t, x, y) & =p_{2}^{D, Y}(x / r, y / r) \\
& \leq c_{8} \mathbb{P}^{y / r}\left(\tau_{D}^{Y}>2\right) \mathbb{P}^{x / r}\left(\tau_{D}^{Y}>2\right) p_{2}^{Y}((x-y) / r) \\
& =c_{8} \mathbb{P}^{y}\left(\tau_{r D}>2 t\right) \mathbb{P}^{y}\left(\tau_{r D}>2 t\right) p_{2 t}(x-y) .
\end{aligned}
$$

Next we deal with the lower bound.

Let $R=\frac{\chi}{\psi^{-1}(1)}$, where $\chi$ is the constant from Proposition 6.4. Recall that

$$
\mathbb{P}^{0}\left(\tau_{(-R / 4, R / 4)}>1\right) \geq 1 / 2 \text {. }
$$

Also note that by Lemma $2.8,1 / C_{1}^{2} \leq V^{2}\left(\frac{1}{\psi^{-1}(1)}\right) \leq V^{2}(R) \leq\left(1+\chi^{2}\right) V^{2}\left(\frac{1}{\psi^{-1}(1)}\right) \leq$ $\left(1+\chi^{2}\right) C_{1}^{2}$. Next, we define for every $z \in D, U_{z}=D \cap(-R, R)$ and $B_{z}=(3 R, 4 R)$ if $|z|<R / 2$ or $U_{z}=(z-R / 4, z+R / 4)$ and $B_{z}=(z+2 R, z+3 R), z>R / 2$ or 
$B_{z}=(z-3 R, z-2 R), z<-R / 2$, otherwise. Note that $V(|w|-1) \geq V(R) \geq 1 / C_{1}$ for $w \in B_{z}$. Hence, by [8, Corollary 3.5] we have

$$
\begin{aligned}
p_{1}^{D}(u, v) & \geq c_{9}(V(|u|-1) \wedge 1)(V(|v|-1) \wedge 1) p_{1}(u-v) \\
& \geq c_{9} C_{1}^{-2} p_{1}(u-v), \quad u \in B_{x}, v \in B_{y} .
\end{aligned}
$$

Moreover it is easy to check that $|u-v| \leq 2((y-x) \vee R)$, hence $p_{1}(u-v) \geq c_{10} p_{1}(x-y)$, which follows from Lemma 6.5. In consequence we have

$$
p_{1}^{D}(u, v) \geq c_{11} p_{1}(x-y), \quad u \in B_{x}, v \in B_{y},
$$

where $c_{11}=c_{9} c_{10} C_{1}^{-2}$. By the semigroup property and Eq. 27 ,

$$
\begin{aligned}
p_{3}^{D}(x, y) & =\int p_{1}^{D}(x, u) p_{1}^{D}(u, v) p_{1}^{D}(v, y) d u d v \\
& \geq c_{11} p_{1}(x-y) \int_{B_{x}} p_{1}^{D}(x, u) d u \int_{B_{y}} p_{1}^{D}(x, v) d v .
\end{aligned}
$$

Next for $u \in B_{x}^{\prime \prime}$ which is an interval with the same center as $B_{x}$, and has the length $\left|B_{x}^{\prime \prime}\right|=$ $\left|B_{x}\right| / 2$, we have by Lemma 6.6

$$
p_{1}^{D}(x, u) \geq \mathbb{P}^{x}\left(\tau_{U_{x}}>1\right) \mathbb{P}^{u}\left(\tau_{B_{x}}>1\right) \inf _{u \in U_{x}, z \in B_{x}} v(z-u) \geq \mathbb{P}^{x}\left(\tau_{U_{x}}>1\right) \mathbb{P}^{u}\left(\tau_{B_{x}}>1\right) v(5 R) .
$$

Next note that, by Eq. 26,

$$
\mathbb{P}^{u}\left(\tau_{B_{x}}>1\right) \geq \mathbb{P}^{0}\left(\tau_{(-R / 4,-R / 4)}>1\right) \geq 1 / 2
$$

and

$$
\mathbb{P}^{x}\left(\tau_{U_{x}}>1\right) \geq \frac{C_{9}}{2} \mathbb{P}^{x}\left(\tau_{D}>1\right),
$$

which follows from Proposition 6.4 for $|x| \leq R / 2$ and from Eq. 26 for $|x|>R / 2$. Hence, using $v(5 R) \approx \frac{1}{R V^{2}(5 R)} \approx \frac{1}{R}$, we arrive at

$$
\int_{B_{x}} p_{1}^{D}(x, u) d u \geq \frac{C_{9}}{4} \mathbb{P}^{x}\left(\tau_{D}>1\right) v(R)\left|B_{x}^{\prime \prime}\right| \geq c_{12} \frac{\left|B_{x}^{\prime \prime}\right|}{R} \mathbb{P}^{x}\left(\tau_{D}>1\right) \geq c_{13} \mathbb{P}^{x}\left(\tau_{D}>1\right),
$$

since $\left|B_{x}^{\prime \prime}\right| \approx R$. The above estimates combined with Eq. 28 yield

$$
p_{D}(3, x, y) \geq c_{11} c_{13}^{2} p_{1}(x-y) \mathbb{P}^{x}\left(\tau_{D}>1\right) \mathbb{P}^{y}\left(\tau_{D}>1\right) .
$$

Finally, by observing that $p_{3}(x-y) \approx p_{1}(x-y)($ Lemma 6.5$)$ and $\mathbb{P}^{x}\left(\tau_{D}>3\right) \approx \mathbb{P}^{x}\left(\tau_{D}>\right.$ 1) (Theorem 5.5), we arrive at

$$
p_{D}(3, x, y) \geq c_{14} \mathbb{P}^{x}\left(\tau_{D}>3\right) p(3, x, y) \mathbb{P}^{y}\left(\tau_{D}>3\right) .
$$

Applying the same scaling argument as used for the upper bound we conclude for any $t, r>0$,

$$
p_{t}^{r D}(x, y) \geq c_{14} \mathbb{P}^{x}\left(\tau_{r D}>t\right) p_{t}(x-y) \mathbb{P}^{y}\left(\tau_{r D}>t\right) .
$$

The proof is completed.

Remark 6 If we consider the semigroup killed upon hitting $\{0\}$ then with the assumptions of Theorem 6.7 we can obtain the following estimate of its transition density

$$
p_{t}^{\{0\}^{c}}(x, y) \approx \mathbb{P}^{x}\left(T_{0}>t\right) \mathbb{P}^{y}\left(T_{0}>t\right) p_{t}(x-y), \quad t>0, \quad x, y \neq 0 .
$$

This can be proved either by taking the limit in the estimates from Theorem 6.7 if $r \rightarrow 0$ or by proving directly following the steps of the proof above. 
Now, we suggest the following extension of Theorem 6.7.

Remark 7 Let $X$ be a pure jump process. The assumption of unimodality of $X$ can be removed by assuming certain estimates of the symmetric Lévy density of $X$. Suppose that

$$
v(x) \approx \frac{f(1 /|x|)}{|x|}, x \in \mathbb{R},
$$

where $f:[0, \infty) \rightarrow[0, \infty)$ is non-decreasing and $f \in \operatorname{WLSC}\left(\alpha, \gamma_{1}\right) \cap \operatorname{WUSC}\left(\beta, \rho_{1}\right)$, $1<\alpha<\beta<2$. Then according to [7, Proposition 28] the characteristic exponent of $X$, $\psi \approx f$, so $\psi \in \operatorname{WUSC}(\beta, \rho) \cap \operatorname{WUSC}(\beta, \rho)$. Moreover, by the result of Chen, $\operatorname{Kim}$ and Kumagai [13] we have

$$
p_{t}(x) \approx f^{-1}(1 / t) \wedge t \frac{f(1 /|x|)}{|x|} \approx p_{t}(0) \wedge t \frac{f(1 /|x|)}{|x|} \approx \psi^{-1}(1 / t) \wedge t v(x) .
$$

We also note that the renewal function of the ladder process is $V_{X}(x) \approx \frac{1}{\sqrt{\psi(1 / x)}} \approx \frac{1}{\sqrt{f(1 / x)}}$. Hence we conclude that Lemma 6.5 holds in this case. Moreover the density $p_{t}(x)$ is almost non-increasing for $x \in(0, \infty)$ that is there is symmetric $q_{t}(x)$ non-increasing on $(0, \infty)$ and a constant $c \geq 1$ such that

$$
c^{-1} q_{t}(x) \leq p_{t}(x) \leq c q_{t}(x), \quad t>0, x \in \mathbb{R} .
$$

Therefore we can repeat, with necesarry slight modifications, all the steps from the proof of Theorem 6.7 and obtain its conclusion in this case. The details are left to interested readers.

Acknowledgments We thank the referees for helpful comments and suggestions.

Open Access This article is distributed under the terms of the Creative Commons Attribution 4.0 International License (http://creativecommons.org/licenses/by/4.0/), which permits unrestricted use, distribution, and reproduction in any medium, provided you give appropriate credit to the original author(s) and the source, provide a link to the Creative Commons license, and indicate if changes were made.

\section{References}

1. Bass, R.F., Levin, D.A.: Harnack inequalities for jump processes. Potential Anal. 17(4), 375-388 (2002)

2. Bertoin, J.: Lévy processes, volume 121 of Cambridge Tracts in Mathematics. Cambridge University Press, Cambridge (1996)

3. Bingham, N.H., Goldie, C.M., Teugels, J.L.: Regular variation, volume 27 of Encyclopedia of Mathematics and its Applications. Cambridge University Press, Cambridge (1989)

4. Blumenthal, R.M., Getoor, R.K.: Markov processes and potential theory Pure and Applied Mathematics, vol. 29. Academic Press, New York-London (1968)

5. Bogdan, K., Grzywny, T., Ryznar, M.: Barriers, exit time and survival probability for unimodal Lévy processes. Probab. Theory Relat. Fields 162, 155-198 (2015)

6. Bogdan, K., Grzywny, T., Ryznar, M.: Heat kernel estimates for the fractional Laplacian with Dirichlet conditions. Ann. Probab. 38(5), 1901-1923 (2010)

7. Bogdan, K., Grzywny, T., Ryznar, M.: Density and tails of unimodal convolution semigroups. J. Funct. Anal. 266(6), 3543-3571 (2014)

8. Bogdan, K., Grzywny, T., Ryznar, M.: Dirichlet heat kernel for unimodal Lévy processes. Stoch. Process Appl. 124(11), 3612-3650 (2014)

9. Bogdan, K., Kumagai, T., Kwaśnicki, M.: Boundary Harnack inequality for Markov processes with jumps. Trans. Amer. Math Soc. 367(1), 477-517 (2015)

10. Bogdan, K., Żak, T.: On Kelvin transformation. J. Theoret. Probab. 19(1), 89-120 (2006)

11. Bretagnolle, J.: Résultats de Kesten sur les processus à accroissements indépendants. In: Séminaire de Probabilités, V (Univ. Strasbourg, année universitaire 1969-1970), pages 21-36. Lecture Notes in Math., vol. 191. Springer, Berlin (1971) 
12. Chen, Z.-Q., Kim, P., Kumagai, T.: On heat kernel estimates and parabolic Harnack inequality for jump processes on metric measure spaces. Acta Math. Sin. (Engl. Ser.) 25(7), 1067-1086 (2009)

13. Chen, Z.-Q., Kim, P., Kumagai, T.: Global heat kernel estimates for symmetric jump processes. Trans. Amer. Math Soc. 363(9), 5021-5055 (2011)

14. Chen, Z.-Q., Kim, P., Song, R.: Heat kernel estimates for the Dirichlet fractional Laplacian. J. Eur. Math Soc. (JEMS) 12(5), 1307-1329 (2010)

15. Chen, Z.-Q., Kim, P., Song, R.: Global heat kernel estimate for relativistic stable processes in exterior open sets. J. Funct. Anal. 263(2), 448-475 (2012)

16. Chen, Z.-Q., Tokle, J.: Global heat kernel estimates for fractional Laplacians in unbounded open sets. Probab. Theory Relat. Fields 149(3-4), 373-395 (2011)

17. Chung, K.L., Zhao, Z.X.: From Brownian motion to Schrödinger's equation, volume 312 of Grundlehren der Mathematischen Wissenschaften [Fundamental Principles of Mathematical Sciences]. SpringerVerlag, Berlin (1995)

18. Doney, R.A.: Hitting probabilities for spectrally positive Lévy processes. J. London Math. Soc. (2) 44(3), 566-576 (1991)

19. Eisenbaum, N., Kaspi, H., Marcus, M.B., Rosen, J., Shi, Z.: A Ray-Knight theorem for symmetric Markov processes. Ann. Probab. 28(4), 1781-1796 (2000)

20. Gorin, E.A., Kukushkin, B.N.: Integrals related to the cantor function. Algebra i Anal. 15(3), 188-220 (2003)

21. Grzywny, T.: On Harnack inequality and Hölder regularity for isotropic unimodal Lévy processes. Potential Anal. 41(1), 1-29 (2014)

22. Grzywny, T., Ryznar, M.: Potential theory of one-dimensional geometric stable processes Colloq Mathematics 129(1), 7-40 (2012)

23. Hoh, W.: Pseudo differential operators generating Markov processes. Habilitationsschrift, Universität Bielefeld (1998)

24. Ikeda, N., Watanabe, S.: On some relations between the harmonic measure and the Lévy measure for a certain class of Markov processes. J. Math. Kyoto Univ. 2, 79-95 (1962)

25. Juszczyszyn, T., Kwaśnicki, M.: Hitting times of points for symmetric Lévy processes with completely monotone jumps. Electron. J Probab. 20(48), 24 (2015)

26. Kim, K.-Y.: Global heat kernel estimates for symmetric Markov processes dominated by stable-like processes in exterior $C^{1}, \eta$ open sets. Potential Anal. 43(2), 127-148 (2015)

27. Kim, P., Song, R., Vondraček, Z.: Global uniform boundary Harnack principle with explicit decay rate and its application. Stoch. Process Appl. 124(1), 235-267 (2014)

28. Kuznetsov, A., Kyprianou, A.E., Pardo, J.C., Watson, A.R.: The hitting time of zero for a stable process. Electron. J. Probab. 19(30), 26 (2014)

29. Kwaśnicki, M.: Spectral theory for symmetric one-dimensional Lévy processes killed upon hitting the origin. Electron. J. Probab. 17(83), 29 (2012)

30. Kwaśnicki, M., Małecki, J., Ryznar, M.: Suprema of Lévy processes. Ann. Probab. 41(3B), 2047-2065 (2013)

31. Peskir, G.: The law of the hitting times to points by a stable Lévy process with no negative jumps. Electron. Commun. Probab. 13, 653-659 (2008)

32. Port, S.C.: Hitting times and potentials for recurrent stable processes. J. Hitting Anal. Math. 20, 371-395 (1967)

33. Pruitt, W.E.: The growth of random walks and Lévy processes. Ann. Probab. 9(6), 948-956 (1981)

34. Sato, K.-I.: Lévy processes and infinitely divisible distributions, volume 68 of Cambridge Studies in Advanced Mathematics. Cambridge University Press, Cambridge (1999). Translated from the 1990 Japanese original, Revised by the author

35. Schilling, R.L., Song, R., Vondraček, Z. Bernstein functions. Theory and applications, volume 37 of de Gruyter Studies in Mathematics, 2nd edn. Walter de Gruyter \& Co., Berlin (2012)

36. Silverstein, M.L.: Classification of coharmonic and coinvariant functions for a Lévy process. Ann. Probab. 8(3), 539-575 (1980)

37. Simon, T.: Hitting densities for spectrally positive stable processes. Stochastics 83(2), 203-214 (2011)

38. Song, R., Vondraček, Z.: Harnack inequality for some classes of Markov processes. Math. Z. 246(1-2), 177-202 (2004)

39. Song, R., Vondraček, Z.: On suprema of Lévy processes and application in risk theory. Ann. Inst. Henri. Poincaré Probab. Stat. 44(5), 977-986 (2008)

40. Watanabe, T.: The isoperimetric inequality for isotropic unimodal Lévy processes. Z. Wahrsch. Verw. Gebiete 63(4), 487-499 (1983) 
41. Yano, K.: Excursions away from a regular point for one-dimensional symmetric Lévy processes without Gaussian part. Potential Anal. 32(4), 305-341 (2010)

42. Yano, K., Yano, Y., Yor, M.: On the laws of first hitting times of points for one-dimensional symmetric stable Lévy processes. In: Séminaire de Probabilités XLII, volume 1979 of Lecture Notes in Mathematics, pp. 187-227. Springer, Berlin (2009) 